\title{
SKEW YOUNG DIAGRAM METHOD IN SPECTRAL DECOMPOSITION OF INTEGRABLE LATTICE MODELS
}

\author{
Anatol N. Kirillov'], Atsuo Kuniba², And Tomoki Nakanishi[ \\ ${ }^{1}$ Department of Mathematical Sciences, University of Tokyo \\ Komaba, Meguro-ku, Tokyo 153, Japan \\ ${ }^{2}$ Institute of Physics, University of Tokyo \\ Komaba, Meguro-ku, Tokyo 153, Japan \\ ${ }^{3}$ Department of Mathematics, University of North Carolina \\ Chapel Hill, NC 27599, USA
}

July, 1996

\begin{abstract}
The spectral decomposition of the path space of the vertex model associated to the vector representation of the quantized affine algebra $U_{q}\left(\widehat{s l}_{n}\right)$ is studied. We give a one-to-one correspondence between the spin configurations and the semistandard tableaux of skew Young diagrams. As a result we obtain a formula of the characters for the degeneracy of the spectrum in terms of skew Schur functions. We conjecture that our result describes the $s l_{n}$-module contents of the Yangian $Y\left(s l_{n}\right)$ module structures of the level 1 integrable modules of the affine Lie algebra $\widehat{s l}_{n}$. An analogous result is obtained also for a vertex model associated to the quantized twisted affine algebra $U_{q}\left(A_{2 n}^{(2)}\right)$, where $Y\left(B_{n}\right)$ characters appear for the degeneracy of the spectrum. The relation to the spectrum of the Haldane-Shastry and the Polychronakos models are also discussed.
\end{abstract}

\footnotetext{
${ }^{1}$ Permanent address: Steklov Mathematical Institute, Fontanka 27, St. Petersburg, 191011, Russia

${ }^{3}$ Permanent address: Department of Mathematics, Nagoya University, Chikusa-ku, Nagoya 464, Japan
} 


\section{INTRODUCTION}

The corner transfer matrix (CTM) has been attracting much attention in the recent study of integrable lattice models based on the Yang-Baxter equation [3]. The CTM acts on the space of paths, which is often identified with the semi-infinite tensor product of a finite-dimensional quantum group module. It is well known that the trace of the CTM of a vertex model associated to the quantized affine algebra $U_{q}(\widehat{g})$ is related to affine Lie algebra characters. We call this correspondence the DJKMO (Date-Jimbo-Kuniba-Miwa-Okado) correspondence.

In [2] a fine structure of the CTM spectrum, called the spectral decomposition, is studied in the $U_{q}\left(\hat{s l}_{2}\right)$ vertex models. The idea behind it is as follows: The logarithmic derivative of the CTM is regarded as the energy operator, or the Hamiltonian, of the path space. As a nature of an integrable system, we expect that there exists a family of commuting operators (the integrals of motion) which act on the path space and commute with the Hamiltonian. The spectral decomposition is the simultaneous diagonalization of these integrals of motion. The degeneracy of the spectrum, then, reflects the non-abelian symmetry which commutes with these integrals of motion.

In the meanwhile, the action of the Yangian algebra $Y\left(s l_{2}\right)$ is defined on the level 1 integrable modules of the untwisted affine Lie algebra $\widehat{s l}_{2}$ in [11], and their $Y\left(s l_{2}\right)$ module structures are determined [6]. It turns out that the degeneracy of the spectrum of the CTM Hamiltonian in the $U_{q}\left(\widehat{s l}_{2}\right)$ vertex model exactly describes the $Y\left(s l_{2}\right)$ module structure of the level 1 integrable modules [2].

We believe this coincidence is a universal phenomena. Namely, we expect that a similar coincidence occurs between the spectrum of the commutant of the non-abelian, probably some quantum group, symmetry of a conformal field theory and the CTM spectrum in the corresponding lattice model.

Motivated by this expectation, in this paper we study the spectral decomposition of the vertex model of the vector representation of the $U_{q}\left(\widehat{s l}_{n}\right)$. The counter part of the DJKMO correspondence is the level 1 integrable modules of $\widehat{s l}_{n}$. The action of the Yangian $Y\left(s l_{n}\right)$ on these modules is defined in [22], but the $Y\left(s l_{n}\right)$-module structure is not fully studied yet (however, see [5] for a related result). In this paper we determine the characters of the degeneracy of the spectrum, and show that they are the characters of irreducible $Y\left(s l_{n}\right)$-modules as expected. Therefore, we conjecture that our spectral decomposition exactly describes the $Y\left(s l_{n}\right)$-module structure of the level 1 integrable modules at the character level.

Conceptually, the spectral decomposition in the $s l_{n}$ case is formulated just as in the case of $s l_{2}$. However, due to the complexity of the irreducible modules of $Y\left(s l_{n}\right)$ for $n \geq 3$, the incidence matrix technique used in [2] is not very efficient. A key to overcome this difficulty is the observation that there exists a natural one-to-one correspondence between the paths of the vertex model and the semi-standard tableaux of certain skew Young diagrams. The appearance of the skew Young diagrams is not 
quite unexpected, because they label a family of $Y\left(s l_{n}\right)$-modules, called the tame modules in 20]. Thanks to this correspondence, we can express the characters of the degeneracy of the spectrum in terms of the skew Schur functions. This enables us to identify them as irreducible $Y\left(s l_{n}\right)$ characters.

It is possible to extend our analysis for a vertex model associated to the quantized twisted affine algebra $U_{q}\left(A_{2 n}^{(2)}\right)$. The characters of the degeneracy of the spectrum are analogues of the skew Schur functions. They are conjectured in [18 to be irreducible $Y\left(B_{n}\right)$ characters.

The content of the paper is as follows: In section 2 we review the DJKMO correspondence for the vertex model in the $s l_{n}$ case. In section 3 we formulate the spectral decomposition of the model. In section 4 some properties of skew Young diagrams and skew Schur functions are given. In section 5 we describe the correspondence between the configurations of the vertex model and the semi-standard tableaux, and determine the characters of the degeneracy of the spectrum. In section 6 the identifications with irreducible $Y\left(g l_{n}\right)$ and $Y\left(s l_{n}\right)$ characters are given. In section 7 we discuss the relation with the spectrum of other type of spin models, such as the Haldane-Shastry model and the Polychronakos model. In section 8 an analogous result for an $U_{q}\left(A_{2 n}^{(2)}\right)$ vertex model is presented. In Appendix A the equality between the skew Schur functions and the Rogers-Szegö polynomials is proved. In Appendix B a new combinatorial description of the Kostka-Foulkes polynomials is given. In Appendix $\mathrm{C}$ we describe the level 1 character of $A_{2 n}^{(2)}$.

\section{DJKMO CORRESPONDENCE}

We review the correspondence between the CTM spectrum of the vertex models of the vector representation of $U_{q}\left(\widehat{s l}_{n}\right)$ and the affine Lie algebra characters of $\widehat{s l}_{n}$ [8].

For given two infinite sequences, $\vec{a}=\left(a_{1}, a_{2}, \ldots\right)$ and $\vec{b}=\left(b_{1}, b_{2}, \ldots\right)$, of any kind of objects $a_{i}, b_{i}$, we write $\vec{a} \approx \vec{b}$ if $a_{i} \neq b_{i}$ only for finitely many $i$. We often use a shorthand notation $\vec{a}=\left(a_{1}, \ldots, a_{k},\left(a_{k+1}, \ldots, a_{k+m}\right)^{\infty}\right)$ for such a periodic sequence as $\vec{a}=\left(a_{1}, \ldots, a_{k}, a_{k+1}, \ldots, a_{k+m}, a_{k+1}, \ldots, a_{k+m}, \ldots\right)$.

Let $\bar{\Lambda}_{1}, \ldots, \bar{\Lambda}_{n-1}$ be the fundamental weights of the Lie algebra $s l_{n}$, and let

$$
\epsilon_{i}=\bar{\Lambda}_{i}-\bar{\Lambda}_{i-1}
$$

for $i=1, \ldots, n$ with $\bar{\Lambda}_{0}=\bar{\Lambda}_{n}=0$. Then $B\left(\bar{\Lambda}_{1}\right)=\left\{\epsilon_{1}, \ldots, \epsilon_{n}\right\}$ is the set of all the weights of the irreducible representation (vector representation) of $s l_{n}$ whose highest weight is $\bar{\Lambda}_{1}$. We give a total ordering in $B\left(\bar{\Lambda}_{1}\right)$ as $\epsilon_{1} \prec \epsilon_{2} \prec \cdots \prec \epsilon_{n}$.

We define the local energy function $H: B\left(\bar{\Lambda}_{1}\right) \times B\left(\bar{\Lambda}_{1}\right) \rightarrow\{0,1\}$ as

$$
H\left(\epsilon_{i}, \epsilon_{j}\right)= \begin{cases}0 & \text { if } \epsilon_{i} \prec \epsilon_{j} \\ 1 & \text { if } \epsilon_{i} \succeq \epsilon_{j}\end{cases}
$$


The function $H$ will play an essential role in our study. It is the logarithm of the $R$-matrix associated to the vector representation of $U_{q}\left(\widehat{s l}_{n}\right)$ in the limit $q \rightarrow 0$. form

An infinite sequence $\vec{s}=\left(s_{i}\right), s_{i} \in B\left(\bar{\Lambda}_{1}\right)$, is called a spin configuration if it has a

$$
\vec{s}=\left(s_{1}, \ldots, s_{m},\left(\epsilon_{1}, \epsilon_{2}, \ldots, \epsilon_{n}\right)^{\infty}\right),
$$

where $s_{1}, \ldots, s_{m}$ is an arbitrary finite sequence. Equivalently, $\vec{s}$ is a spin configuration if $\vec{s} \approx \vec{s}^{(k)}$ for some $k=0, \ldots, n-1$, where

$$
\vec{s}^{(k)}=\left(\epsilon_{1}, \epsilon_{2}, \ldots, \epsilon_{k},\left(\epsilon_{1}, \epsilon_{2}, \ldots, \epsilon_{n}\right)^{\infty}\right) .
$$

The set of all the spin configurations $\mathcal{S}$ has a natural decomposition

$$
\mathcal{S}=\bigsqcup_{k=0}^{n-1} \mathcal{S}^{(k)}, \quad \mathcal{S}^{(k)}=\left\{\vec{s} \mid \vec{s} \approx \vec{s}^{(k)}\right\}
$$

For $\vec{s}=\left(s_{i}\right) \in \mathcal{S}^{(k)}$ we define its energy $E(\vec{s})$ and $s l_{n}$-weight $\operatorname{wt}(\vec{s})$ as

$$
\begin{aligned}
E(\vec{s}) & =\sum_{i=1}^{\infty} i\left\{H\left(s_{i}, s_{i+1}\right)-H\left(s_{i}^{(k)}, s_{i+1}^{(k)}\right)\right\}, \\
\operatorname{wt}(\vec{s}) & =\bar{\Lambda}_{k}+\sum_{i=1}^{\infty}\left(s_{i}-s_{i}^{(k)}\right) .
\end{aligned}
$$

Proposition 2.1. Let $\vec{s}=\left(s_{1}, \ldots, s_{m},\left(\epsilon_{1}, \ldots, \epsilon_{n}\right)^{\infty}\right)$ be any element of $\mathcal{S}^{(k)}$. Then

$$
\mathrm{wt}(\vec{s})=\sum_{i=1}^{m} s_{i} \text {. }
$$

Proof. Since $m \equiv k$ modulo $n, \sum_{i=1}^{m} s_{i}^{(k)}=\bar{\Lambda}_{k}$. Thus

$$
\sum_{i=1}^{m} s_{i}=\bar{\Lambda}_{k}+\sum_{i=1}^{m}\left(s_{i}-s_{i}^{(k)}\right)=\bar{\Lambda}_{k}+\sum_{i=1}^{\infty}\left(s_{i}-s_{i}^{(k)}\right)=\operatorname{wt}(\vec{s}) .
$$

There is a remarkable connection between the partition function of $\mathcal{S}^{(k)}$ and an affine Lie algebra character.

Theorem 2.2 (DJKMO correspondence [8, 15]). For $k=0,1, \ldots, n-1$, let $\mathcal{L}\left(\Lambda_{k}\right)$ be the level 1 integrable module of the untwisted affine Lie algebra $\widehat{s l}_{n}$ whose highest weight is the kth fundamental weight $\Lambda_{k}$ of $\widehat{s l}_{n}$. Then the following equality holds:

$$
\begin{aligned}
\operatorname{ch} \mathcal{L}\left(\Lambda_{k}\right) & =q^{\Delta_{k}-c / 24} \sum_{\vec{s} \in \mathcal{S}^{(k)}} q^{E(\vec{s})} e^{\mathrm{wt}(\vec{s})} \\
& =q^{\Delta_{k}-c / 24} \sum_{\vec{s} \in \mathcal{S}^{(n-k)}} q^{E(\vec{s})} e^{-\mathrm{wt}(\vec{s})},
\end{aligned}
$$


where $\operatorname{ch} \mathcal{L}\left(\Lambda_{k}\right), \Delta_{k}=k(n-k) / 2 n$, and $c=n-1$ are the (normalized) character, the conformal dimension, and the Virasoro central charge of $\mathcal{L}\left(\Lambda_{k}\right)$, respectively [13. In (2.6b) $\mathcal{S}^{(n)}=\mathcal{S}^{(0)}$.

Remark . Often spin configurations are described by an alternative notion, paths. An infinite sequence $\vec{p}=\left(p_{i}\right)$ of $\widehat{s l}_{n}$-weights is called a path if it satisfies the conditions: (i) $p_{i+1}-p_{i} \in B\left(\bar{\Lambda}_{1}\right)$, (ii) $\vec{p}=\left(p_{1}, \ldots, p_{m},\left(\Lambda_{0}, \Lambda_{1}, \ldots, \Lambda_{n-1}\right)^{\infty}\right)$. It is clear that the map $\vec{p}=\left(p_{i}\right) \mapsto \vec{s}=\left(p_{i+1}-p_{i}\right)$ is a bijection from the set of all the paths $\mathcal{P}$ to $\mathcal{S}$. By this identification our spin configurations are also called paths in some literature. The $s l_{n}$-weight of a path $\vec{p}=\left(p_{i}\right)$ is defined as $\operatorname{wt}(\vec{p})=\overline{p_{1}}$. Then, under the bijection $\vec{p} \mapsto \vec{s}, \operatorname{wt}(\vec{p})=-\mathrm{wt}(\vec{s})$ holds. It is this context where $(2.6 \mathrm{~b})$ is proved in $[8]$. The expression (2.6a) follows from (2.6b) by the Dynkin diagram automorphism $\alpha_{i} \leftrightarrow \alpha_{n-i}$ of $s l_{n}$.

\section{Spectral DeComposition}

We introduce the local energy map $h: \mathcal{S} \rightarrow\{0,1\}^{\mathbf{N}}$ such that

$$
h: \vec{s}=\left(s_{i}\right) \mapsto \vec{h}=\left(h_{i}\right), \quad h_{i}=H\left(s_{i}, s_{i+1}\right) .
$$

Each number $h_{i}$ is called the $i$ th local energy of $\vec{s}$. We call the image $\operatorname{Sp}=h(\mathcal{S})$ the spectrum of $\mathcal{S}$. Let $\mathrm{Sp}^{(k)}=h\left(\mathcal{S}^{(k)}\right)$. Then we have the decomposition $\mathrm{Sp}=\bigsqcup_{k=0}^{n-1} \mathrm{Sp}^{(k)}$. The element of $\mathrm{Sp}^{(k)}$ is characterized as follows.

Proposition 3.1. An element $\vec{h}=\left(h_{i}\right) \in\{0,1\}^{\mathbf{N}}$ belongs to $\mathrm{Sp}^{(k)}$ if and only if it satisfies the conditions,

$$
\begin{aligned}
& \text { (i) } h_{i}+h_{i+1}+\cdots+h_{i+n-1} \geq 1 \text { for any } i \text {. } \\
& \text { (ii) } \vec{h} \approx \vec{h}^{(k)} \text {, where } \vec{h}^{(k)}:=h\left(\vec{s}^{(k)}\right)=(\underbrace{0, \ldots, 0,1}_{k},(\underbrace{0, \ldots, 0,1}_{n})^{\infty}) \text {. }
\end{aligned}
$$

One can paraphrase the condition (3.2a) as "There are at most $n-1$ consecutive 0 's in $\vec{h}$ ". Here we prove only the necessity of the conditions. The sufficiency will be proved after Prop. 5.1 in section 5 .

Proof. Let us assume $h_{i}=h_{i+1}=\cdots=h_{i+n-2}=0$. Then we have $s_{i} \prec s_{i+1} \prec \cdots \prec$ $s_{i+n-1}$, from which $s_{i+n-1}=\epsilon_{n}$ follows. Therefore, $h_{i+n-1}=H\left(s_{i+n-1}, s_{i+n}\right)=1$ regardless of the value of $s_{i+n}$.

The condition (ii) is an immediate consequence of (2.3).

Any element $\vec{h}$ of $\mathrm{Sp}^{(k)}$ is uniquely written in the form

$$
\left[m_{1}, \ldots, m_{r}\right]:=(\underbrace{0, \ldots, 0,1}_{m_{1}}, \ldots, \underbrace{0, \ldots, 0,1}_{m_{r}},(\underbrace{0, \ldots, 0,1}_{n})^{\infty}), \quad 1 \leq m_{i} \leq n, m_{r} \neq n \text {. }
$$


Obviously

$$
\mathrm{Sp}^{(k)}=\left\{\left[m_{1}, \ldots, m_{r}\right] \mid r \geq 0,1 \leq m_{i} \leq n, m_{r} \neq n, \sum_{i=1}^{r} m_{i} \equiv k \quad \bmod n\right\} .
$$

The surjection $h: \mathcal{S}^{(k)} \rightarrow \mathrm{Sp}^{(k)}$ induces the decomposition of $\mathcal{S}^{(k)}$,

$$
\mathcal{S}^{(k)}=\bigsqcup_{\vec{h} \in \mathrm{Sp}^{(k)}} \mathcal{S}_{\vec{h}}, \quad \mathcal{S}_{\vec{h}}=h^{-1}(\vec{h})
$$

We call this decomposition the spectral decomposition of $\mathcal{S}^{(k)}$.

Let us introduce the character of the degeneracy of the spectrum at $\vec{h}$,

$$
\begin{aligned}
\operatorname{ch} \mathcal{S}_{\vec{h}} & =q^{\Delta_{k}-c / 24} \sum_{\vec{s} \in \mathcal{S}_{\vec{h}}} q^{E(\vec{s})} e^{\mathrm{wt}(\vec{s})} \\
& =q^{\Delta_{k}-c / 24+\sum_{i=1}^{\infty} i\left(h_{i}-h_{i}^{(k)}\right)} \chi_{\vec{h}}, \quad \chi_{\vec{h}}=\sum_{\vec{s} \in \mathcal{S}_{\vec{h}}} e^{\mathrm{wt}(\vec{s})}
\end{aligned}
$$

As is standard in the character theory of $s l_{n}$, we regard $\chi_{\vec{h}}=\chi_{\vec{h}}(x)$ as a function of the variables $x_{1}=e^{\epsilon_{1}}, x_{2}=e^{\epsilon_{2}}, \ldots, x_{n}=e^{\epsilon_{n}}$ with the relation $x_{1} x_{2} \cdots x_{n}=1$. Due to Theorem 2.2, the character of $\mathcal{L}\left(\Lambda_{k}\right)$ is decomposed as

$$
\operatorname{ch} \mathcal{L}\left(\Lambda_{k}\right)(q, x)=q^{\Delta_{k}-c / 24} \sum_{\vec{h} \in \mathrm{Sp}^{(k)}} q^{\sum_{i=1}^{\infty} i\left(h_{i}-h_{i}^{(k)}\right)} \chi_{\vec{h}}(x) .
$$

The main purpose of the paper is to calculate the characters $\chi_{\vec{h}}$ and to show that they are irreducible characters of $Y\left(s l_{n}\right)$. To do it, we make use of a hidden relation between spin configurations and skew Young diagrams.

\section{SkeW Diagrams And SKeW Schur FunCtions}

Let us recall the definitions of a skew diagram, a semi-standard tableau, and the skew Schur function. We basically follow the definitions and notations of [19].

A partition $\lambda=\left(\lambda_{1}, \lambda_{2}, \ldots, \lambda_{m}\right)$ is a non-increasing sequence of non-negative integers, $\lambda_{1} \geq \lambda_{2} \geq \cdots \geq \lambda_{m} \geq 0$. We let $|\lambda|=\sum_{i=1}^{m} \lambda_{i}$. The length $l(\lambda)$ of $\lambda$ is the number of the non-zero elements in $\lambda$. As usual, a partition $\lambda$ is represented by a (Young) diagram, which is denoted by the same symbol $\lambda$. We conveniently identify the partitions $\left(\lambda_{1}, \ldots, \lambda_{m}\right),\left(\lambda_{1}, \ldots, \lambda_{m}, 0\right),\left(\lambda_{1}, \ldots, \lambda_{m}, 0,0\right)$, etc. The conjugate of a partition $\lambda$ is a partition $\lambda^{\prime}$ whose diagram is the transpose of the diagram of $\lambda$ along the main diagonal. For example, if $\lambda=(4,3,2)$, then its conjugate is $\lambda^{\prime}=(3,3,2,1)$.

For a pair of partitions $\lambda$ and $\mu$, we write $\lambda \supset \mu$ if $\lambda_{i} \geq \mu_{i}$ for any $i$. If $\lambda \supset \mu$, the diagram $\mu$ is naturally embedded inside the diagram $\lambda$. Then the skew (Young) diagram $\lambda / \mu$ (denoted by $\lambda-\mu$ in [19]) is obtained by subtracting the diagram $\mu$ from the diagram $\lambda$. For example, if $\lambda=(5,4,4,1)$ and $\mu=(4,3,2,0)$, then $\lambda / \mu$ looks as 


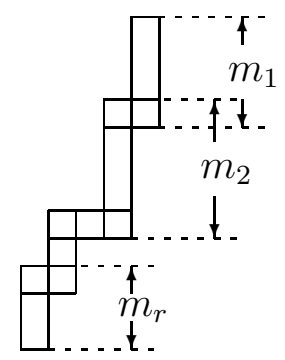

FiguRE 1. A border strip $\left\langle m_{1}, \ldots, m_{r}\right\rangle$.

follows:

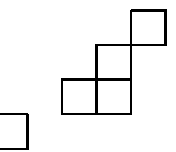

We set $|\lambda / \mu|=|\lambda|-|\mu|$.

We say $\lambda / \mu$ is a skew diagram of rank $n$ if the length of any column of $\lambda / \mu$ does not exceed $n$. Two boxes in a skew diagram are adjacent if they share a common side. A skew diagram $\lambda / \mu$ is connected if, for any pair of boxes $a$ and $a^{\prime}$ in $\lambda / \mu$, there exits a series of boxes $b_{1}=a, b_{2}, \ldots, b_{j}=a^{\prime}$ in $\lambda / \mu$ such that $b_{i}$ and $b_{i+1}$ are adjacent. A skew diagram is called a border strip if it is connected, and contains no $2 \times 2$ block of boxes. Let $\left\langle m_{1}, \ldots, m_{r}\right\rangle$ denote the border strip of $r$ columns such that the length of $i$ th column (from the right) is $m_{i}$ (Fig. 1).

For a skew diagram $\lambda / \mu$, we now define the skew Schur function $s_{\lambda / \mu}$. In each box of a given skew diagram $\lambda / \mu$, let us inscribe one of the numbers $1,2, \ldots, n$. We call such an arrangement of numbers a semi-standard tableau $T$ of shape $\lambda / \mu$, if it satisfies the following condition: Let $a$ and $b$ be the inscribed numbers in any pair of adjacent boxes. Then,

$$
\begin{aligned}
& \text { (i) } a<b \text { if } b \text { is lower-adjacent to } a \text {. } \\
& \text { (ii) } a \geq b \text { if } b \text { is left-adjacent to } a \text {. }
\end{aligned}
$$

The $s l_{n}$-weight of a semi-standard tableau $T$ is defined as

$$
\mathrm{wt}^{\prime}(T)=\sum_{a=1}^{n} m_{a} \cdot \epsilon_{a}
$$

where $m_{a}$ is the number counting how many $a$ 's are in $T$, and $\epsilon_{a}$ is given in (2.1).

Definition 4.1. The skew Schur function $s_{\lambda / \mu}$ is defined as

$$
s_{\lambda / \mu}(x)=\sum_{T \in \operatorname{SST}(\lambda / \mu)} e^{\mathrm{wt}^{\prime}(T)}, \quad x_{i}=e^{\epsilon_{i}},
$$


where $\operatorname{SST}(\lambda / \mu)$ is the set of all the semi-standard tableaux of shape $\lambda / \mu$.

The following proposition is well-known. See section 5 of [19] for a proof.

Proposition 4.1. The skew Schur function $s_{\lambda / \mu}$ is also expressed as

$$
s_{\lambda / \mu}(x)=\operatorname{det}\left(e_{\lambda_{i}^{\prime}-\mu_{j}^{\prime}-i+j}(x)\right)_{1 \leq i, j \leq r},
$$

where $r \geq l\left(\lambda^{\prime}\right)$, and $e_{m}=e_{m}(x)$ is the $m$ th elementary symmetric polynomial of variables $x_{1}, \ldots, x_{n}$ for $m=0, \ldots, n$, and $e_{m}=0$ for other $m$.

We impose the relation $x_{1} x_{2} \cdots x_{n}=1$ throughout the paper. Then $e_{m}$ is the character of the $m$ th fundamental representation of $s l_{n}$ with the highest weight $\bar{\Lambda}_{m}$ for $m=1, \ldots, n-1$.

The following properties of $s_{\lambda / \mu}$ follow either from (4.3) or from (4.4):

(i) If $\lambda / \mu$ is not a skew diagram of rank $n$, then $s_{\lambda / \mu}=0$.

(ii) When $\mu=(0)$, the expression (4.4) reduces to the Jacobi-Trudi formula of the ordinary Schur function $s_{\lambda}$.

(iii) Let $c_{\mu \nu}^{\lambda},|\lambda|=|\mu|+|\nu|$, be the Littlewood-Richardson coefficient, i.e., $s_{\mu} s_{\nu}=$ $\sum_{\lambda} c_{\mu \nu}^{\lambda} s_{\lambda}$. Then, $s_{\lambda / \mu}=\sum_{\nu} c_{\mu \nu}^{\lambda} s_{\nu}$.

The conjugate $s_{\lambda / \mu}^{*}$ of the skew Schur function $s_{\lambda / \mu}$ is defined as

$$
\begin{aligned}
s_{\lambda / \mu}^{*} & =\operatorname{det}\left(e_{n-\lambda_{i}^{\prime}+\mu_{j}^{\prime}+i-j}\right)_{1 \leq i, j \leq r} \\
& =\sum_{T \in \operatorname{SST}(\lambda / \mu)} e^{-\mathrm{wt}^{\prime}(T)} .
\end{aligned}
$$

It is also possible to express $s_{\lambda / \mu}^{*}$ as a skew Schur function. Suppose $\lambda / \mu$ is of rank $n$ with $\mu=\left(\mu_{1}, \ldots, \mu_{m}\right)$. Then we have a new pair

$$
\tilde{\mu}=(\underbrace{\lambda_{1}, \ldots, \lambda_{1}}_{n}, \mu_{1}, \ldots, \mu_{m}) \supset \lambda,
$$

and the compliment of $\lambda / \mu,(\lambda / \mu)^{c}:=\tilde{\mu} / \lambda$, is also a skew diagram of rank $n$. The picture below illustrates the example of $n=4, \lambda=(5,4,3,1), \mu=(3,2)$ :

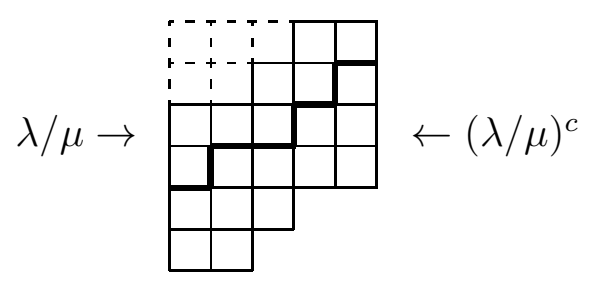

Proposition 4.2. Let $\lambda / \mu$ be a skew diagram of rank $n$. Then

$$
\begin{gathered}
s_{\lambda / \mu}^{*}=s_{(\lambda / \mu)^{c}} . \\
\text { Proof. } s_{(\lambda / \mu)^{c}}=\operatorname{det}\left(e_{n+\mu_{i}^{\prime}-\lambda_{j}^{\prime}-i+j}\right)=\operatorname{det}\left(e_{n-\lambda_{i}^{\prime}+\mu_{j}^{\prime}+i-j}\right)=s_{\lambda / \mu}^{*} .
\end{gathered}
$$




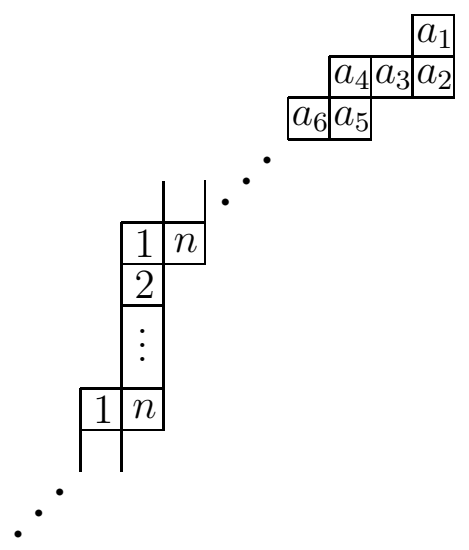

Figure 2. A semi-standard tableau $\tilde{T}$ of shape $\tilde{\kappa}(\vec{h})$ for $\vec{h}=$ $\left(0,1,1,0,1, \ldots,(0, \ldots, 0,1)^{\infty}\right)$.

\section{Correspondence BetweEn SPin CONFigurations AND SEMI-STANDARD} TABLEAUX

We proceed to calculate the character $\chi_{\vec{h}}$ of $(3.5)$ using the language of skew diagrams and tableaux.

For a given $\vec{h} \in \mathrm{Sp}$, we associate a skew diagram $\tilde{\kappa}(\vec{h})$ of infinite-size in the following procedure:

1. Write the first box.

2. Attach the second box under (resp. left to) the first box if $h_{1}=0$ (resp. $\left.h_{1}=1\right)$.

3. Similarly attach the $i+1$ th box under (resp. left to) the $i$ th box if $h_{i}=0$ (resp. $h_{i}=1$ ) for $i=2,3, \ldots$

Then $\tilde{\kappa}(\vec{h})$ has the following properties.

(i) It is a border strip.

(ii) It is of rank $n$, i.e., the length of any column of $\tilde{\kappa}(\vec{h})$ does not exceed $n$, due to (3.2a).

(iii) Due to $(3.2 \mathrm{~b})$, it has a periodic tail which consists of length- $n$ columns.

Equivalently, for $\vec{h}=\left[m_{1}, \ldots, m_{r}\right], \tilde{\kappa}(\vec{h})$ is the border strip $\left\langle m_{1}, \ldots, m_{r}, n, n, \ldots\right\rangle$.

A semi-standard tableau $\tilde{T}$ of shape $\tilde{\kappa}(\vec{h})$ is an arrangement of numbers $1, \ldots, n$ in the boxes of $\tilde{\kappa}(\vec{h})$ obeying the conditions (4.1a) and (4.1b), just in the same way as in the finite-size case. See Fig. 2. Notice that the arrangements in the length- $n$ columns are uniquely determined, or "frozen", because of the semi-standard condition (4.1a). Since $\tilde{\kappa}(\vec{h})$ is a border strip, one can give a total ordering of the boxes in it from the right to the left and from the top to the bottom in the unique way. Let

$$
\left(a_{i}\right)=\left(a_{1}, a_{2}, \ldots,(1,2, \ldots, n)^{\infty}\right) .
$$


be the sequence of the content of $\tilde{T}$ along the total ordering of the boxes. Now we have a natural map $\tilde{\varphi}$ from the set of the semi-standard tableaux of shape $\tilde{\kappa}(\vec{h})$ to the set of the spin configurations $\mathcal{S}$ defined by

$$
\tilde{\varphi}(\tilde{T}):=\left(\epsilon_{a_{i}}\right)=\left(\epsilon_{a_{1}}, \epsilon_{a_{2}}, \ldots,\left(\epsilon_{1}, \epsilon_{2}, \ldots, \epsilon_{n}\right)^{\infty}\right) .
$$

A key observation of this paper is

Proposition 5.1. The map $\tilde{\varphi}$ gives a one-to-one correspondence between the semistandard tableaux of shape $\tilde{\kappa}(\vec{h})$ and the spin configurations in $\mathcal{S}_{\vec{h}}$.

Proof. A necessary and sufficient condition for a sequence $\left(a_{1}, a_{2}, \ldots\right), a_{i} \in\{1, \ldots, n\}$ to be the sequence of the content of a semi-standard tableau of shape $\tilde{\kappa}(\vec{h})$ is

$$
a_{i+1}>a_{i}, \quad\left(\text { resp. } a_{i+1} \leq a_{i}\right) \Longleftrightarrow h_{i}=0,\left(\text { resp. } h_{i}=1\right) .
$$

This is an immediate consequence of the construction of $\tilde{\kappa}(\vec{h})$ and $(4.1 \mathrm{a}, \mathrm{b})$. On the other hand (5.2) is also a necessary and sufficient condition for a sequence $\left(\epsilon_{a_{1}}, \epsilon_{a_{2}}, \ldots\right)$ to belong to $\mathcal{S}_{\vec{h}}$ because of (2.2), (3.1), and (3.4).

The first application of Prop. 5.1 is to prove the sufficiency part of Prop. 3.1.

Proof. By Prop. 5.1 we have only to show that there exists at least one semi-standard tableau of shape $\tilde{\kappa}(\vec{h})$ for any $\vec{h}$. In fact, for a given $\vec{h}$, a semi-standard tableau of shape $\tilde{\kappa}(\vec{h})$ is obtained by filling the boxes by $1,2,3, \ldots$ from the top to the bottom in each column. This completes the proof of Prop. 3.1.

For our purpose it is convenient to define a "finite part" $\kappa(\vec{h})$ of the infinite diagram $\tilde{\kappa}(\vec{h})$ by cutting off its periodic tail. Namely, for $\vec{h}=\left[m_{1}, \ldots, m_{r}\right]$ we define $\kappa(\vec{h})=$ $\left\langle m_{1}, \ldots, m_{r}\right\rangle$. (For $\vec{h}=\vec{h}^{(0)}, \kappa\left(\vec{h}^{(0)}\right)$ is the empty diagram $\emptyset$.) It is clear that the map $\kappa: \vec{h} \mapsto \kappa(\vec{h})$ is injective, and we get the following description of the space of the spectrum $\mathrm{Sp}$ in terms of border strips.

Theorem 5.2. The space $\mathrm{Sp}$ is parametrized by the border strips of rank $n$ such that the lengths of their leftmost columns are less than $n$.

The following lemma is obvious.

Lemma 5.3. There is a one-to-one correspondence between the semi-standard tableaux of shape $\tilde{\kappa}(\vec{h})$ and the ones of shape $\kappa(\vec{h})$. The correspondence is given by the restriction of a semi-standard tableau of shape $\tilde{\kappa}(\vec{h})$ on $\kappa(\vec{h})$.

Combining the bijection in Lemma 5.3 with the bijection $\tilde{\varphi}$ of (5.1), we obtain a bijection

$$
\varphi: \operatorname{SST}(\kappa(\vec{h})) \rightarrow \mathcal{S}_{\vec{h}}
$$


Proposition 5.4. The bijection $\varphi: \operatorname{SST}(\kappa(\vec{h})) \rightarrow \mathcal{S}_{\vec{h}}$ is weight-preserving, i.e., for any $T \in \operatorname{SST}(\kappa(\vec{h}))$, wt $(\varphi(T))=\mathrm{wt}^{\prime}(T)$ holds.

Proof. Let $\left(a_{1}, \ldots, a_{m}\right)$ be the content of $T$ aligned along our total order of the boxes in $\kappa(\vec{h})$. Then $\varphi(T)=\left(\epsilon_{a_{1}}, \ldots, \epsilon_{a_{m}},\left(\epsilon_{1}, \ldots, \epsilon_{n}\right)^{\infty}\right)$. From Prop. 2.1 and (4.2), we have $\operatorname{wt}(\varphi(T))=\sum_{i=1}^{m} \epsilon_{a_{i}}=\mathrm{wt}^{\prime}(T)$.

Now we state the first half of our main theorem.

Theorem 5.5. (i) The character $\chi_{\vec{h}}$ of $\mathcal{S}_{\vec{h}}$ is equal to the skew Schur function $s_{\kappa(\vec{h})}$. (ii) Let $\vec{h}=\left[m_{1}, \ldots, m_{r}\right] \in \mathrm{Sp}$. Then

$$
s_{\kappa(\vec{h})}=s_{\left\langle m_{1}, \ldots, m_{r}\right\rangle}=\left|\begin{array}{llllll}
e_{m_{r}} & e_{m_{r}+m_{r-1}} & & \cdots & & e_{m_{r}+\cdots+m_{1}} \\
1 & e_{m_{r-1}} & & & & \\
0 & 1 & & & \\
& 0 & & & \vdots \\
& & \ddots & \ddots & & \\
& & 0 & 1 & e_{m_{2}} & e_{m_{2}+m_{1}} \\
& & & 0 & 1 & e_{m_{1}}
\end{array}\right| .
$$

(iii) The character of the level 1 integrable module $\mathcal{L}\left(\Lambda_{k}\right)$ of $\widehat{s l}_{n}$ decomposes as

$$
\begin{aligned}
\operatorname{ch} \mathcal{L}\left(\Lambda_{k}\right)(q, x) & =q^{-\frac{1}{24} c} \sum_{\substack{\kappa \in B S \\
|\kappa| \equiv k \bmod n}} q^{\frac{1}{2 n}|\kappa|(n-|\kappa|)+t(\kappa)} s_{\kappa}(x) \\
& =q^{-\frac{1}{24} c} \sum_{\substack{\kappa \in B S \\
|\kappa| \equiv n-k \bmod n}} q^{\frac{1}{2 n}|\kappa|(n-|\kappa|)+t(\kappa)} s_{\kappa^{c}}(x),
\end{aligned}
$$

where $B S$ is the set of all the border strips $\kappa=\left\langle m_{1}, \ldots, m_{r}\right\rangle$ of rank $n$ with $m_{r}<n$, and $t(\kappa)=\sum_{i=1}^{r-1}(r-i) m_{i}$.

Proof. The property (i) is an immediate consequence of Prop. 5.4. To show (ii), notice that the skew diagram $\left\langle m_{1}, \ldots, m_{r}\right\rangle$ is represented as $\lambda / \mu$ with a pair $\lambda \supset \mu$ such that

$$
\lambda_{i}^{\prime}=m_{1}+\cdots+m_{r+1-i}-r+i, \quad \mu_{i}^{\prime}=m_{1}+\cdots+m_{r-i}-r+i .
$$

Substituting them into (4.4) we obtain the formula (5.3). The property (iii) follows from (3.6), Prop. 4.2, the property (i), and the fact that for $\vec{h}=\left[m_{1}, \ldots, m_{r}\right] \in \mathrm{Sp}^{(k)}$

$$
\Delta_{k}+\sum_{i=1}^{\infty} i\left(h_{i}-h_{i}^{(k)}\right)=\frac{1}{2 n} m(n-m)+\sum_{i=1}^{r-1}(r-i) m_{i}, \quad m=\sum_{i=1}^{r} m_{i} .
$$

Two expressions (5.4a) and (5.4b) differ from each other when $n \geq 3$. 


\section{YANGIAN CHARACTERS}

In this section we show, based on the result of [20], that the characters $\chi_{\vec{h}}=s_{\kappa(\vec{h})}$ are irreducible characters of the Yangian algebras $Y\left(g l_{n}\right)$ and $Y\left(s l_{n}\right)$.

The Yangian of $g l_{n}, Y\left(g l_{n}\right)$, is an algebra generated by $t_{i j}(r), i, j=1, \ldots, n, r \in \mathbf{Z}_{\geq 0}$ with the relations

$$
\left[t_{i j}(r), t_{k l}(s-1)\right]-\left[t_{i j}(r-1), t_{k l}(s)\right]=t_{k j}(s-1) t_{i l}(r-1)-t_{k j}(r-1) t_{i l}(s-1),
$$

where $t_{i j}(-1)=\delta_{i j} 1, t_{i j}(-2)=0$. The elements $t_{i j}(0)$ generate the universal enveloping algebra of $g l_{n}$. Therefore $s l_{n}$ acts on $Y\left(g l_{n}\right)$-modules.

Consider a pair of partitions $\lambda \supset \mu$ with $\lambda=\left(\lambda_{1}, \ldots, \lambda_{N+n}\right), \mu=\left(\mu_{1}, \ldots, \mu_{N}\right)$, $N \geq 1$. Let $V_{\lambda}$ be the irreducible $g l_{N+n}$-module associated to $\lambda, V_{\mu}$ be the irreducible $g l_{N}$-module associated to $\mu$, and $V_{\lambda, \mu}$ be the space of the multiplicity of $V_{\mu}$ in $V_{\lambda}$ under the standard embedding $g l_{N} \subset g l_{N+n}$. There is an irreducible action of $Y\left(g l_{n}\right)$ on the space $V_{\lambda, \mu}$, having a remarkable property (A module with such property is called a tame module [20]):

Proposition 6.1 ([7, 20]). A maximal commutative subalgebra of $Y\left(g l_{n}\right)$, called the Gelfand-Zetlin (GZ) algebra, acts on $V_{\lambda, \mu}$ in a semi-simple way. Furthermore, a basis diagonalizing the GZ algebra is labeled by the GZ schemes of $V_{\lambda, \mu}$.

A GZ scheme $\backslash \Lambda /$ of $V_{\lambda, \mu}$ is an array of integers $\lambda_{m i}$,

$$
\begin{aligned}
& \begin{array}{lllllllll}
\lambda_{n 1} & \lambda_{n 2} & \cdots & \lambda_{n, N+n} & \lambda_{1} & \lambda_{2} & \cdots & \lambda_{N+n}
\end{array} \\
& \begin{array}{llllll}
\lambda_{n-1,1} & \cdots & \lambda_{n-1, N+n-1} & \lambda_{n-1,1} & \cdots & \lambda_{n-1, N+n-1}
\end{array}
\end{aligned}
$$

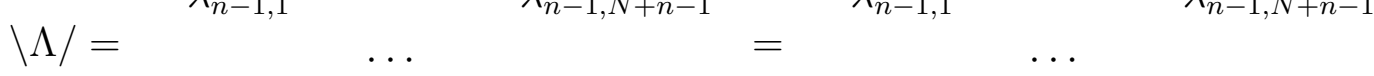

$$
\begin{aligned}
& \begin{array}{llllll}
\lambda_{01} & \cdots & \lambda_{0 N} & \mu_{1} & \cdots & \mu_{N}
\end{array}
\end{aligned}
$$

satisfying the condition $\lambda_{m i} \geq \lambda_{m-1, i} \geq \lambda_{m, i+1}$. The $s l_{n}$-weight of the basis vector labeled by $\backslash \Lambda /$ is

$$
\sum_{m=1}^{n}\left\{\sum_{i=1}^{N+m} \lambda_{m i}-\sum_{i=1}^{N+m-1} \lambda_{m-1, i}\right\} \epsilon_{m} .
$$

Lemma 6.2. There is a weight-preserving, one-to-one correspondence between the GZ schemes of $V_{\lambda, \mu}$ and the semi-standard tableaux of shape $\lambda / \mu$.

The correspondence is described as follows. For a given GZ scheme $\backslash \Lambda /$, we have a sequence of partitions,

$$
\lambda^{(0)}=\mu \subset \lambda^{(1)} \subset \lambda^{(2)} \subset \cdots \subset \lambda^{(n)}=\lambda, \quad \lambda^{(m)}=\left(\lambda_{m 1}, \lambda_{m 2}, \ldots, \lambda_{m, N+m}\right) .
$$


A semi-standard tableau of shape $\lambda / \mu$ is obtained by inscribing the number $m$ in $\lambda^{(m)} / \lambda^{(m-1)}$, which is a part of the diagram $\lambda / \mu$. For example,

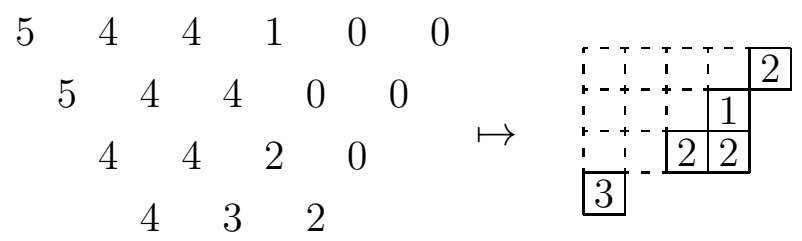

It is easy to check that this map is bijective and weight-preserving.

It follows from Prop. 6.1 and Lemma 6.2 that the $s l_{n}$-character of $V_{\lambda, \mu}$ is equal to $s_{\lambda / \mu}$. In particular, we have

Theorem 6.3. The character $\chi_{\vec{h}}=s_{\kappa(\vec{h})}$ is the sl$l_{n}$-character of the irreducible $Y\left(g l_{n}\right)$ module $V_{\lambda, \mu}$ with $\lambda / \mu=\kappa(\vec{h})$.

The Yangian of $s l_{n}, Y\left(s l_{n}\right)$, is generated by $x_{i k}^{ \pm}, h_{i k}, i=1, \ldots, n-1, k \in \mathbf{Z}_{\geq 0}$ with the relations

$$
\begin{aligned}
& {\left[h_{i k}, h_{j l}\right]=0, \quad\left[h_{i 0}, x_{j l}^{ \pm}\right]= \pm A_{i j} x_{j l}^{ \pm}, \quad\left[x_{i k}^{+}, x_{j l}^{-}\right]=\delta_{i j} h_{i k+l},} \\
& {\left[h_{i k+1}, x_{j l}^{ \pm}\right]-\left[h_{i k}, x_{j l+1}^{ \pm}\right]= \pm \frac{1}{2} A_{i j}\left(h_{i k} x_{j l}^{ \pm}+x_{j l}^{ \pm} h_{i k}\right),} \\
& {\left[x_{i k+1}^{ \pm}, x_{j l}^{ \pm}\right]-\left[x_{i k}^{ \pm}, x_{j l+1}^{ \pm}\right]= \pm \frac{1}{2} A_{i j}\left(x_{i k}^{ \pm} x_{j l}^{ \pm}+x_{j l}^{ \pm} x_{i k}^{ \pm}\right),} \\
& \sum_{\sigma: \text { permutation }}\left[x_{i k_{\sigma(1)}}^{ \pm},\left[x_{i k_{\sigma(2)}}^{ \pm}, \ldots,\left[x_{i k_{\sigma\left(1-A_{i j}\right.}}^{ \pm}, x_{j l}^{ \pm}\right] \ldots\right]\right]=0,
\end{aligned}
$$

where $A_{i j}$ is the Cartan matrix of $s l_{n}$. An irreducible finite-dimensional module of $Y\left(s l_{n}\right)$ is characterized by $n-1$ monic polynomials (the Drinfel'd polynomials), $P_{1}(u), \ldots, P_{n-1}(u)$. The polynomial $P_{i}(u)$ describes the action of $h_{i}(u)=1+$ $\sum_{k=0}^{\infty} h_{i k} u^{-k-1}$ on a highest weight vector $v$ as $h_{i}(u) v=\left(P_{i}(u+1) / P_{i}(u)\right) v$ [9].

Proposition 6.4 ([20]). There is a one-parameter family of irreducible $Y\left(s l_{n}\right)$-module structures on $V_{\lambda, \mu}$ with a parameter $b \in \mathbf{C}$, whose Drinfel'd polynomials are [20]

$$
P_{i}(u)=\prod_{\substack{j=1 \\ \lambda_{j}^{\prime}-\mu_{j}^{\prime}=i}}^{\lambda_{1}}\left(u+\frac{1}{2}\left(\lambda_{j}^{\prime}+\mu_{j}^{\prime}\right)-j+\frac{1}{2}+b\right) .
$$

These $Y\left(s l_{n}\right)$-module structures on $V_{\lambda, \mu}$ are the ones induced by a one-parameter family of embeddings of $Y\left(s l_{n}\right)$ into $Y\left(g l_{n}\right)$. There is a simple pictorial interpretation of the zeros of $P_{i}(u)$ as shown in Fig. 3 .

As a corollary of Prop. 6.4, we obtain the second half of our main theorem:

\footnotetext{
${ }^{1}$ The Drinfel'd polynomials here are the one in [9]. The convention in [20] is slightly different. Their $P_{i}(u)$ is equal to $(-1)^{\operatorname{deg} P_{i}} P_{i}(-u-n / 4+i / 2)$ here.
} 


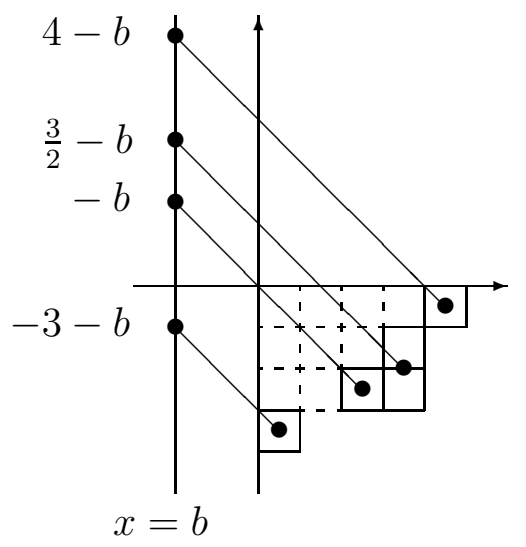

Figure 3. An example: $\lambda=(5,4,4,1), \mu=(4,3,2,0)$. The Drinfel'd polynomials are $P_{1}(u)=(u+3+b)(u+b)(u-4+b), P_{2}(u)=u-\frac{3}{2}+b$, and $P_{i}(u)=1$ for $i \geq 3$. The zeros of $P_{i}(u)$ are identified with the intersections of the line $x=b$ and the diagonal lines passing through the middle points of the columns of length $i$.

Theorem 6.5. The character $\chi_{\vec{h}}=s_{\kappa(\vec{h})}$ is the $l_{n}$-character of the one-parameter family of the irreducible $Y\left(s l_{n}\right)$-modules whose Drinfel'd polynomials are given by (6.1) with $\lambda / \mu=\kappa(\vec{h})$.

We have shown that the characters $\chi_{\vec{h}}$ of the degeneracy of the spectrum are irreducible $Y\left(s l_{n}\right)$ characters. Furthermore, the $Y\left(s l_{n}\right)$-module structure of $\mathcal{L}\left(\Lambda_{k}\right)$ partially studied in [22] agrees with (5.4a). Based on these strong evidences, we conjecture that

Conjecture 6.6. The decomposition (5.4a) describes the $Y\left(s l_{n}\right)$-module structure on $\mathcal{L}\left(\Lambda_{k}\right)$ of 22].

\section{The RELATION With THE SPECTRUM IN OTHER SPIN MODELS}

7.1. The Haldane-Shastry model. The $s l_{n}$ Haldane-Shastry (HS) model is a lattice model with the Hamiltonian

$$
\mathcal{H}_{\mathrm{HS}}=\sum_{1 \leq j \neq k \leq N} \frac{x_{j} x_{k}}{\left(x_{j}-x_{k}\right)\left(x_{k}-x_{j}\right)}\left(P_{j k}-1\right), \quad x_{j}=e^{2 \pi \sqrt{-1} j / N}
$$

acting on the vector space $V^{\otimes N}, V=\mathbf{C}^{n}$, where $P_{j k}$ exchanges the $j$ th and $k$ th components of $V^{\otimes N}$. There is an action of $Y\left(s l_{n}\right)$ on $V^{\otimes N}$ which commutes with the Hamiltonian $\mathcal{H}_{\mathrm{HS}}$ [11]. By this action the space $V^{\otimes N}$ decomposes as [5]

$$
V^{\otimes N} \simeq \bigoplus_{d \in M_{N}} W_{d}
$$


where $W_{d}$ are certain irreducible $Y\left(s l_{n}\right)$ modules described below. $M_{N}$ is the set of the binary sequences $d=\left(d_{1}, \ldots, d_{N-1}\right), d_{i} \in\{0,1\}$, such that there are at most $n-1$ consecutive 1's. The eigenvalue of $\mathcal{H}_{\mathrm{HS}}$ on the eigenspace $W_{d}$ is given by $\sum_{i=1}^{N-1} i d_{i}\left(i d_{i}-N\right)$.

We notice that the condition for $d \in M_{N}$ turns into (3.2a) through the identification $h_{i}=1-d_{i}$. I For a given $d \in M_{N}$, let

$$
\vec{h}_{d}=\left(1-d_{1}, 1-d_{2}, \ldots, 1-d_{N-1}, 1,(\underbrace{0, \ldots, 0,1}_{n})^{\infty}\right) \in \mathrm{Sp} .
$$

We translate the description of the $Y\left(s l_{n}\right)$-module structure of $W_{d}$ in [5] into our language as follows:

Proposition 7.1 ([5]). As a $Y\left(s l_{n}\right)$-module, the eigenspace $W_{d}$ is isomorphic to the irreducible module whose Drinfel'd polynomials are given by (6.1), where in (6.1) $\lambda_{1}$ and $b$ are certain constants independent of $d$, and $\lambda / \mu=\kappa\left(\vec{h}_{d}\right)$.

Comparing Prop. 7.1 with Prop. 6.4 and Theorem 6.5, we obtain the following proposition, which answers the questions of the $s l_{n}$-module content of $W_{d}$ and its factorizability asked in [5, 10, 11, 12].

Proposition 7.2. Let $\vec{h}_{d}=\left[m_{1}, \ldots, m_{r}\right]$. Then

(i) $\operatorname{ch} W_{d}=s_{\left\langle m_{1}, \ldots, m_{r}\right\rangle}$.

(ii) $s_{\left\langle m_{1}, \ldots, m_{r}\right\rangle}=s_{\left\langle m_{1}, \ldots, m_{i}\right\rangle} s_{\left\langle m_{i+1}, \ldots, m_{r}\right\rangle}$ if $m_{i}+m_{i+1} \geq n+1$.

Proof. We only need to prove the property (ii), which follows from (5.3).

7.2. The Polychronakos model. There is another relevant spin model, called the Polychronakos model. The $s l_{n}$ Polychronakos model has the Hamiltonian

$$
\begin{aligned}
\mathcal{H}_{\mathrm{P}} & =\sum_{1 \leq j<k \leq N} \frac{1}{\left(x_{j}-x_{k}\right)^{2}}\left(P_{j k}-1\right)+E_{N}, \\
E_{N} & =\frac{n-1}{2 n} N^{2}-\frac{\bar{N}(n-\bar{N})}{2 n},
\end{aligned}
$$

acting on $V^{\otimes N}, V=\mathbf{C}^{n}$, where $x_{1}, \ldots, x_{N}$ are the zeros of the Hermite polynomial of degree $N$, and $\bar{N} \equiv N \bmod n, 0 \leq \bar{N} \leq n-1$. The constant $E_{N}$ is added to make the ground state energy zero. Define the partition function of the Polychronakos model

$$
Z_{N}^{\mathrm{P}}(q, x)=\operatorname{tr}_{V \otimes N} q^{\mathcal{H} \mathrm{P}} \prod_{i=1}^{n-1} x_{i}^{h_{i}+\cdots+h_{n-1}}
$$

\footnotetext{
${ }^{2}$ This intriguing relation between the spectrum of the HS model and the vertex model was first indicated by Bernard [4] in the $s l_{2}$ case.
} 
where $h_{i}$ 's are the standard basis of the Cartan subalgebra of $s l_{n}$. It is shown in [21] that

$$
Z_{N}^{\mathrm{P}}(q, x)=q^{E_{N}} H_{N}\left(q^{-1}, x\right)
$$

where $H_{N}(q, x)$ is a generalization of the Rogers-Szegö polynomial [1],

$$
H_{N}(q, x)=\sum_{\substack{k_{i} \in \mathbf{Z}_{\geq 0} \\ k_{1}+\cdots+k_{n}=N}} \frac{(q)_{N}}{(q)_{k_{1}}(q)_{k_{2}} \cdots(q)_{k_{n}}} x_{1}^{k_{1}} \cdots x_{n}^{k_{n}}, \quad(q)_{k}=\prod_{i=1}^{k}\left(1-q^{i}\right) .
$$

Again there is an action of $Y\left(s l_{n}\right)$ on $V^{\otimes N}$, which commutes with $\mathcal{H}_{\mathrm{P}}$ [12]. Based on a numerical study, it was conjectured in [12] that

1. As a $Y\left(s l_{n}\right)$-module, the spin space $V^{\otimes N}$ decomposes exactly in the same way as in (7.1).

2. The eigenvalue $E_{d}$ of $\mathcal{H}_{\mathrm{P}}$ on $W_{d}$ is

$$
E_{d}=-\sum_{i=1}^{N-1} i d_{i}+E_{N}
$$

Let us introduce the sets,

$$
\begin{aligned}
\operatorname{Sp}_{N} & =\left\{\vec{h} \in \operatorname{Sp}^{(\bar{N})} \mid h_{i}=h_{i}^{(\bar{N})} \text { for } i \geq N\right\}, \\
\mathcal{S}_{N} & =\left\{\vec{s} \in \mathcal{S}^{(\bar{N})} \mid s_{i}=s_{i}^{(\bar{N})} \text { for } i \geq N+1\right\} .
\end{aligned}
$$

Lemma 7.3. Let $N$ be a positive integer. Then

(i) The map $d \mapsto \vec{h}_{d}$ is a bijection from $M_{N}$ to $\mathrm{Sp}_{N}$.

(ii) $h^{-1}\left(\operatorname{Sp}_{N}\right)=\mathcal{S}_{N}$.

(iii) For any $d \in M_{N}$ and $\vec{s} \in \mathcal{S}_{\vec{h}_{d}}, E_{d}=E(\vec{s})$.

(iv) The $s l_{n}$-character of $W_{d}$ is $s_{\kappa\left(\vec{h}_{d}\right)}=\chi_{\vec{h}_{d}}$.

We define

$$
Z_{N}^{\mathrm{vertex}}(q, x)=\sum_{\vec{s} \in \mathcal{S}_{N}} q^{E(\vec{s})} e^{\mathrm{wt}(\vec{s})}
$$

Then Lemma 7.3 and the above conjecture of [12] claims the identification,

$$
Z_{N}^{\mathrm{P}}=Z_{N}^{\mathrm{vertex}}
$$

This exact equivalence of two spectra is intriguing, because the Hamiltonian for $Z_{N}^{\text {vertex }}$ is of nearest-neighborhood type, while $\mathcal{H}_{P}$ is not. In fact, the following theorem proves (7.11) directly, thereby providing a further support for the conjecture of [12].

Theorem 7.4. For any nonnegative integer $N$, we have

$$
\sum_{\vec{h} \in \operatorname{Sp}_{N}} q^{\sum_{i=1}^{\infty} i\left(h_{i}-h_{i}^{(\bar{N})}\right)} s_{\kappa(\vec{h})}(x)=q^{E_{N}} H_{N}\left(q^{-1}, x\right) .
$$


A proof of Theorem 7.4 is given in Appendix A.

As a corollary of Theorem 7.4 we get an expression

$$
q^{\Delta_{k}-c / 24} \sum_{\vec{s} \in \mathcal{S}^{(k)}} q^{E(\vec{s})} e^{\mathrm{wt}(\vec{s})}=\lim _{\substack{N \rightarrow \infty \\ N \equiv k \bmod n}} q^{\Delta_{k}-c / 24+E_{N}} H_{N}\left(q^{-1}, x\right) .
$$

The right hand side of (7.12) converges to (cf. [12, 21])

$$
\frac{1}{\eta(q)^{n-1}} \sum_{\substack{k_{i} \in \mathbf{Z}-\frac{k}{n} \\ k_{1}+\cdots+k_{n}=0}} q^{\frac{1}{2} k_{1}^{2}+\cdots+\frac{1}{2} k_{n}^{2}} x_{1}^{k_{1}} \cdots x_{n}^{k_{n}}, \quad \eta(q)=q^{\frac{1}{24}}(q)_{\infty}
$$

which is equal to

$$
\frac{1}{\eta(q)^{n-1}} \sum_{\gamma \in \oplus_{i=1}^{n-1} \mathbf{Z} \alpha_{i}} q^{\frac{1}{2}\left|\bar{\Lambda}_{k}+\gamma\right|^{2}} e^{\bar{\Lambda}_{k}+\gamma}
$$

where $\alpha_{i}$ 's, $\left|\alpha_{i}\right|^{2}=2$, are the simple roots of $s l_{n}$. The series (7.13) is a well-known expression of $\operatorname{ch} \mathcal{L}\left(\Lambda_{k}\right)$ [13]. Therefore we have obtained an alternative proof of Theorem 2.2 .

As another corollary of Theorem 7.4, a new combinatorial description of the KostkaFoulkes polynomials is obtained. See Appendix B.

\section{VERTEX MODEL OF $U_{q}\left(A_{2 n}^{(2)}\right)$}

One can apply the skew diagram method also to a vertex model associated to the quantized twisted affine algebra $U_{q}\left(A_{2 n}^{(2)}\right)$ [17 with an appropriate modification. We expect that the underlying algebra for the degeneracy is the Yangian of $B_{n}, Y\left(B_{n}\right)$.

In contrast with the standard textbook [13], we regard $A_{2 n}^{(2)}$ as an affinization of the Lie algebra $B_{n}$ rather than $C_{n}$. Their Dynkin diagrams are depicted in Fig. 4 . Let $\bar{\Lambda}_{i}(i=1, \ldots, n)$ be the fundamental weights of $B_{n}$, and let $\epsilon_{ \pm i}= \pm\left(\bar{\Lambda}_{i}-\bar{\Lambda}_{i-1}\right)$ for $i=1, \ldots, n-1, \epsilon_{ \pm n}= \pm\left(2 \bar{\Lambda}_{n}-\bar{\Lambda}_{n-1}\right)$, and $\epsilon_{0}=0$. Then $B\left(\bar{\Lambda}_{1}\right)=\left\{\epsilon_{1} \prec \cdots \prec\right.$ $\left.\epsilon_{n} \prec \epsilon_{0} \prec \epsilon_{-n} \prec \cdots \prec \epsilon_{-1}\right\}$ is the set of all the weights of the vector representation of $B_{n}$ with a total ordering $\prec$. The local energy function $H: B\left(\bar{\Lambda}_{1}\right) \times B\left(\bar{\Lambda}_{1}\right) \rightarrow\{0,1\}$ is defined as

$$
H\left(s, s^{\prime}\right)= \begin{cases}0 & \text { if } s \prec s^{\prime} \text { or }\left(s, s^{\prime}\right)=\left(\epsilon_{0}, \epsilon_{0}\right), \\ 1 & \text { if } s \succeq s^{\prime} \text { and }\left(s, s^{\prime}\right) \neq\left(\epsilon_{0}, \epsilon_{0}\right) .\end{cases}
$$

A sequence $\vec{s}=\left(s_{i}\right), s_{i} \in B\left(\bar{\Lambda}_{1}\right)$, is a spin configuration if $\vec{s} \approx\left(\left(\epsilon_{0}\right)^{\infty}\right)$. Let $\mathcal{S}$ be the set of all the spin configurations. For each $\vec{s} \in \mathcal{S}$ we define

$$
E(\vec{s})=\sum_{i=1}^{\infty} i H\left(s_{i}, s_{i+1}\right), \quad \operatorname{wt}(\vec{s})=-\bar{\Lambda}_{n}+\sum_{i=1}^{\infty} s_{i} .
$$




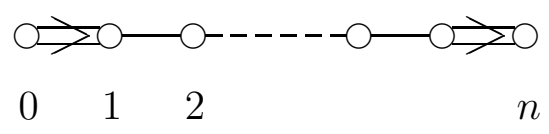

Figure 4. The Dynkin diagram of $A_{2 n}^{(2)}$. The Dynkin diagram of $B_{n}$ is obtained by removing the node 0 .

Theorem 8.1 (15]). Let $\operatorname{ch} \mathcal{L}\left(\Lambda_{n}\right)$ be the unnormalized character of the (unique) level 1 integrable module of $A_{2 n}^{(2)}$. Then

$$
\operatorname{ch} \mathcal{L}\left(\Lambda_{n}\right)(q, x)=\sum_{\vec{s} \in \mathcal{S}} q^{E(\vec{s})} e^{\mathrm{wt}(\vec{s})}, \quad e^{\epsilon_{ \pm i}}=x_{i}^{ \pm 1}, e^{\epsilon_{0}}=1 .
$$

See Appendix $\square$ for the explicit expression of $\operatorname{ch} \mathcal{L}\left(\Lambda_{n}\right)(q, x)$.

The local energy map $h: \vec{s} \mapsto \vec{h}=\left(h_{i}\right), h_{i}=H\left(s_{i}, s_{i+1}\right)$, has the image $h(\mathcal{S})=\mathrm{Sp}$, where $\operatorname{Sp}=\left\{\vec{h} \mid \vec{h} \approx\left((0)^{\infty}\right)\right\}$. Any element $\vec{h} \in \mathrm{Sp}$ is written in the form

$$
\left[m_{1}, \ldots, m_{r}\right]:=(\underbrace{0, \ldots, 0,1}_{m_{1}}, \ldots, \underbrace{0, \ldots, 0,1}_{m_{r}},(0)^{\infty}), \quad m_{i} \geq 1
$$

We set $\mathcal{S}_{\vec{h}}=h^{-1}(\vec{h})$ for $\vec{h} \in \mathrm{Sp}$, and define $\chi_{\vec{h}}(x)=\sum_{\vec{s} \in \mathcal{S}_{\vec{h}}} e^{\operatorname{wt}(\vec{s})}$.

For each $\vec{h} \in \mathrm{Sp}$, we associate a skew diagram $\tilde{\kappa}(\vec{h})$ of infinite-size, following the procedures $1-3$ in the beginning of section 5. Namely, if $\vec{h}=\left[m_{1}, \ldots, m_{r}\right]$, then $\tilde{\kappa}(\vec{h})$ is a border strip with $r+1$ columns, $\left\langle m_{1}, \ldots, m_{r}, \infty\right\rangle$. Also we define a border strip $\kappa(\vec{h})=\left\langle m_{1}, \ldots, m_{r}, 2 n\right\rangle$ as a "finite part" of $\tilde{\kappa}(\vec{h})$.

An analogue of the semi-standard condition suited for $Y\left(B_{n}\right)$ is introduced in [18] to characterize the spectrum of the row-to-row transfer matrices with a wide class of auxiliary spaces. We find that the notion in $[18$ are quite adequate also for the description of $\chi_{\vec{h}}$. Let $J=\{1 \prec \cdots \prec n \prec 0 \prec-n \prec \cdots \prec-1\}$. We inscribe the numbers from $J$ in each box of a skew diagram $\lambda / \mu$. We call such an arrangement an admissible tableau of shape $\lambda / \mu$ if it satisfies the following condition: Let $a$ and $b$ be the inscribed numbers in any pair of adjacent boxes. Then,

(i) $a \prec b$ or $(a, b)=(0,0)$ if $b$ is lower-adjacent to $a$.

(ii) $a \succeq b$ and $(a, b) \neq(0,0)$ if $b$ is left-adjacent to $a$.

Let $\operatorname{AT}(\lambda / \mu)$ be the set of all the admissible tableaux of shape $\lambda / \mu$. Since the condition $(8.4 \mathrm{a}, \mathrm{b})$ is just in coordinate with (8.1), there is a natural bijection between $\mathcal{S}_{\vec{h}}$ and $\operatorname{AT}(\tilde{\kappa}(\vec{h}))$ as in section 5. However, the set $\operatorname{AT}(\kappa(\vec{h}))$ is larger than $\operatorname{AT}(\tilde{\kappa}(\vec{h}))$. Therefore we introduce a further constraint on the elements in $\operatorname{AT}(\kappa(\vec{h}))$. An admissible tableau of shape $\kappa(\vec{h})$ is $L($ eft $)$-admissible if the content of the bottom $n$ boxes 
in the leftmost column is frozen to the sequence $-n,-n+1, \ldots,-1$. 3 We write the set of all the L-admissible tableaux of shape $\kappa(\vec{h})$ as $\operatorname{LAT}(\kappa(\vec{h}))$. Then

Lemma 8.2. There is a bijection from $\operatorname{AT}(\tilde{\kappa}(\vec{h}))$ to $\operatorname{LAT}(\kappa(\vec{h}))$.

The correspondence is a natural one: For $\tilde{T} \in \operatorname{AT}(\tilde{\kappa}(\vec{h}))$, let $\left(a_{1}, \ldots, a_{m},(0)^{\infty}\right)$ be its content, where $a_{m-n+1}, \ldots, a_{m}$ are in the top $n$ boxes in the leftmost column. Then, $\left(a_{1}, \ldots, a_{m},-n, \ldots,-1\right)$ gives the content of the corresponding tableau $T \in$ $\operatorname{LAT}(\kappa(\vec{h}))$. Combining the two bijections, we obtain a bijection $\varphi: \operatorname{LAT}(\kappa(\vec{h})) \rightarrow \mathcal{S}_{\vec{h}}$.

For an L-admissible tableau $T \in \operatorname{LAT}(\kappa(\vec{h}))$ with the content $\left(a_{1}, \ldots, a_{m},-n, \ldots,-1\right)$, its weight is defined as $\mathrm{wt}^{\prime}(T)=\sum_{i=1}^{m} \epsilon_{a_{i}}+\frac{1}{2} \sum_{i=1}^{n} \epsilon_{-i}=\sum_{i=1}^{m} \epsilon_{a_{i}}-\bar{\Lambda}_{n}$, where, following [18], we multiply the factor $\frac{1}{2}$ on the weights corresponding to the bottom $n$ boxes in the leftmost column of $\kappa(\vec{h})$. Comparing it with $(8.2)$, we see that the bijection $\varphi: \operatorname{LAT}(\kappa(\vec{h})) \rightarrow \mathcal{S}_{\vec{h}}$ is weight-preserving. Therefore we have

Theorem 8.3. (i) The character $\chi_{\vec{h}}$ of $\mathcal{S}_{\vec{h}}$ is equal to

$$
s_{\kappa(\vec{h})}^{\mathrm{L}}:=\sum_{T \in \operatorname{LAT}(\kappa(\vec{h}))} e^{\mathrm{wt}^{\prime}(T)} .
$$

(ii) Let $\vec{h}=\left[m_{1}, \ldots, m_{r}\right] \in \mathrm{Sp}$. Then

$$
s_{\kappa(\vec{h})}^{\mathrm{L}}=s_{\left\langle m_{1}, \ldots, m_{r}, 2 n\right\rangle}^{\mathrm{L}}=\sigma\left|\begin{array}{llllll}
1 & 1 & & \cdots & 1 \\
1 & t_{m_{r}} & t_{m_{r}+m_{r-1}} & & & t_{m_{r}+\cdots+m_{1}} \\
0 & 1 & t_{m_{r-1}} & & & \\
& 0 & 1 & & \vdots \\
& & & & \ddots & \\
& & & 1 & t_{m_{2}} & t_{m_{2}+m_{1}} \\
& & & 0 & 1 & t_{m_{1}}
\end{array}\right|,
$$

where $\sigma$ is $\operatorname{ch} V_{\bar{\Lambda}_{n}}, t_{m}$ is 0 for $m<0$, ch $\left(V_{\bar{\Lambda}_{m}} \oplus V_{\bar{\Lambda}_{m-2}} \oplus \cdots\right)$ for $0 \leq m \leq n-1$, and $\sigma^{2}-t_{2 n-1-m}$ for $m \geq n$, and $V_{\bar{\Lambda}_{j}}$ is the $j$ th fundamental representation of $B_{n}$.

(iii) The character of the level 1 integrable module $\mathcal{L}\left(\Lambda_{n}\right)$ of $A_{2 n}^{(2)}$ decomposes as

$$
\operatorname{ch} \mathcal{L}\left(\Lambda_{n}\right)(q, x)=\sum_{\kappa \in B S} q^{t(\kappa)} s_{\kappa}^{\mathrm{L}}(x),
$$

where $B S$ is the set of all the border strips $\kappa=\left\langle m_{1}, \ldots, m_{r}\right\rangle$ with $m_{r}=2 n$, and $t(\kappa)=\sum_{i=1}^{r-1}(r-i) m_{i}$.

Proof. (i) and (iii) are due to the bijection $\varphi$ and Theorem 8.1. (ii) is a special case of Theorem 4.1 in [18, which is an analogue of Prop. 4.1 for $s_{\lambda / \mu}^{\mathrm{L}}$.

\footnotetext{
${ }^{3}$ The definition of the L-admissibility here is the simplified one especially for a border strip with the length of the last column $2 n$. See 18 for the definition for a general L-hatched skew diagram.
} 
It was conjectured in [18] that $s_{\kappa(\vec{h})}^{\mathrm{L}}$ is the $B_{n}$-character of a certain irreducible $Y\left(B_{n}\right)$-module. Therefore it is natural to conjecture that

Conjecture 8.4. There is a canonical action of $Y\left(B_{n}\right)$ on the level 1 integrable module $\mathcal{L}\left(\Lambda_{n}\right)$ of $A_{2 n}^{(2)}$. The decomposition (8.5) describes its $Y\left(B_{n}\right)$-module structure on $\mathcal{L}\left(\Lambda_{n}\right)$.

\section{Conclusion}

In this paper we exhibit intimate relationships among the spectral decomposition of the vertex models, skew diagrams and the associated Schur functions, irreducible characters of the Yangians, Yangian module structures in conformal field theory, spectra of spin models with the inverse-square interaction, and so on. We believe that further study of this interrelation will enlighten our understanding of the common integrable structure behind these models.

It is also interesting to investigate other vertex models. For example, for the symmetric fusion models of $U_{q}\left(\widehat{s l}_{n}\right)$, which correspond to the higher level integrable modules of $\widehat{s l}_{n}$, it is possible to extend our skew diagram approach. Even though conceptually it is quite analogous to the level 1 case, some complexity enters, especially for $n \geq 3$. Notable changes are, firstly, skew diagrams of non-border strips are necessary to describe the spectrum, and secondly, the characters of non-tame modules appear as the characters of the degeneracy of the spectrum. We hope to give a full report on it in a future publication.

At the very last stage of the preparation of the manuscript, the preprint "The $\widehat{S U}(n)_{1}$ WZW models, spinon decomposition and Yangian structure" by P. Bouwknegt and K. Schoutens (hep-th/9607064) appeared, where the authors obtain a partially overlapping result to ours.

Acknowledgment. A. N. K. would like to thank the colleagues from Tokyo University for kind hospitality and support. A. K. and T. N. appreciate the great hospitality of the organizers of the Third International Conference on Conformal Filed Theory and Integrable Models held at Landau Institute of Theoretical Physics, Chernogolovka on June 24-29, 1996, where the main result of this paper is presented. T. N. would like to thank G. Felder and A. Varchenko for their warm hospitality at University of North Carolina.

\section{Appendix A. A proof of Theorem 7.4}

In this appendix we give a proof of Theorem 7.4 .

Any element of $\vec{h} \in \mathrm{Sp}_{N}$ is uniquely written as

$$
\vec{h}=(\underbrace{0, \ldots, 0,1}_{m_{1}}, \underbrace{0, \ldots, 0,1}_{m_{2}}, \ldots, \underbrace{0, \ldots, 0,1}_{m_{r}},(\underbrace{0, \ldots, 0,1}_{n})^{\infty})
$$


for an integer $r$ (= the number of 1's in the first $N$ elements of $\vec{h}$ ) and the integers $1 \leq m_{i} \leq n$ such that $m_{1}+m_{2}+\cdots+m_{r}=N$. For such an $\vec{h} \in \mathcal{S}_{N}$ we associate a border strip $\left\langle m_{1}, \ldots, m_{r}\right\rangle$. If $m_{r} \neq n$, the border strip $\left\langle m_{1}, \ldots, m_{r}\right\rangle$ is equal to $\kappa(\vec{h})$. If $m_{r}=n$, however, $\left\langle m_{1}, \ldots, m_{r}\right\rangle$ has a few more length- $n$ columns than $\kappa(\vec{h})$ on its tail such that the size of $\left\langle m_{1}, \ldots, m_{r}\right\rangle$ is always $N$. In either case, we have $s_{\kappa(\vec{h})}=s_{\left\langle m_{1}, \ldots, m_{r}\right\rangle}$ thanks to the specialization $x_{1} \cdots x_{n}=1$. Furthermore, for $\vec{h} \in \operatorname{Sp}_{N}$ it holds that

$$
\sum_{i=1}^{\infty} i\left(h_{i}-h_{i}^{(\bar{N})}\right)=\sum_{i=1}^{N-1} i\left(h_{i}-1\right)+E_{N}=\sum_{i=1}^{N} i\left(h_{i}-1\right)+E_{N} .
$$

Thus Theorem 7.4 is equivalent to

Theorem A.1.

$$
\sum_{r=1}^{N} \sum_{\substack{1 \leq m_{i} \leq n \\ m_{1}+\cdots+m_{r}=N}} q^{\frac{1}{2} N(N+1)-\sum_{i=1}^{r}\left(m_{1}+\cdots+m_{i}\right)} s_{\left\langle m_{1}, \ldots, m_{r}\right\rangle}(x)=H_{N}(q, x) .
$$

Remark. Theorem A.1 is true without assuming the relation $x_{1} \cdots x_{n}=1$ as we show below.

We write the left hand side of (A.1) as $F_{N}(q, x)\left(F_{0}(q, x)=1\right.$ by definition). Example.

$$
\begin{array}{ll}
F_{0}(q, x)=1, & H_{0}(q, x)=1, \\
F_{1}(q, x)=s_{\square}(x), & H_{1}(q, x)=\sum_{i=1}^{n} x_{i}, \\
F_{2}(q, x)=q s_{\text {日 }}(x)+s_{\text {Ш }}(x), & H_{2}(q, x)=\sum_{i=1}^{n} x_{i}^{2}+(1+q) \sum_{1 \leq i<j \leq n} x_{i} x_{j} .
\end{array}
$$

Following [12], we consider the recursion relation for $F_{N}(q, x)$ and $H_{N}(q, x)$. Let

$$
G(q, x, t)=\frac{1}{\left(t x_{1} ; q\right)_{\infty}\left(t x_{2} ; q\right)_{\infty} \cdots\left(t x_{n} ; q\right)_{\infty}}, \quad(a ; q)_{\infty}=\prod_{j=0}^{\infty}\left(1-a q^{j}\right) .
$$

Lemma A.2 (匹1). The function $G(q, x, t)$ is the generating function of $H_{N}(q, x)$ :

$$
G(q, x, t)=\sum_{N=0}^{\infty} \frac{H_{N}(q, x)}{(q)_{N}} t^{N}
$$

Proof. It easily follows from the identity [1]

$$
\frac{1}{(t ; q)_{\infty}}=\sum_{N=0}^{\infty} \frac{1}{(q)_{N}} t^{N}
$$


Lemma A.3 (匹1, 12]). The functions $H_{N}(q, x)$ satisfy the following recursion relation: For any $N \geq 1$,

$$
H_{N}(q, x)=\sum_{i=1}^{n}(-1)^{i+1} \frac{(q)_{N-1}}{(q)_{N-i}} e_{i}(x) H_{N-i}(q, x)=0
$$

where $e_{i}(x)$ is the $i$ th elementary symmetric function of $x_{1}, \ldots, x_{n}$, and $H_{N}(q, x)=0$ for $N<0$.

Proof. Consider the identity,

$$
G(q, x, q t)=\left(\prod_{i=1}^{n}\left(1-t x_{i}\right)\right) G(q, x, t)=\left(\sum_{i=0}^{n}(-1)^{i} e_{i}(x) t^{i}\right) G(q, x, t) .
$$

Comparing the coefficients of $t^{N}$ of the both sides, and using Lemma A.2, we have

$$
\frac{q^{N}}{(q)_{N}} H_{N}(q, x)=\sum_{i=0}^{n}(-1)^{i} e_{i}(x) \frac{H_{N-i}(q, x)}{(q)_{N-i}}
$$

from which the lemma follows.

The recursion relation (A.2), together with the initial condition $H_{0}(q, x)=1$, uniquely determines $H_{N}(q, x)$. In the rest of the appendix we show $F_{N}(q, x)$ also satisfies (A.2). We recall

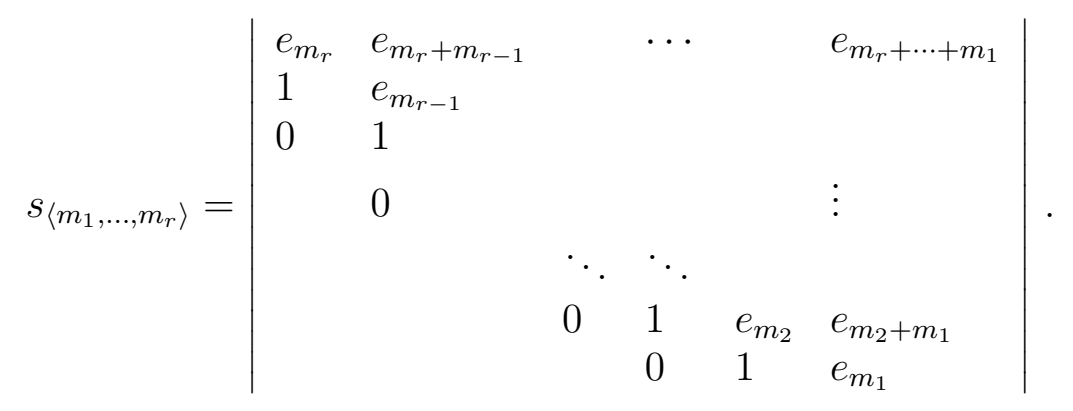

Expanding the determinant along the first row, we have

\section{Lemma A.4.}

$$
s_{\left\langle m_{1}, \ldots, m_{r}\right\rangle}=\sum_{i=1}^{r}(-1)^{i+1} e_{m_{r}+\cdots+m_{r-i+1}} s_{\left\langle m_{1}, \ldots, m_{r-i}\right\rangle} .
$$

Substituting (A.3) into $F_{N}(q, x)$, we have 
Lemma A.5. The functions $F_{N}(q, x)$ satisfy the following recursion relation: For any $N \geq 1$,

$$
\begin{aligned}
F_{N}(q, x)= & \sum_{m=1}^{N} A_{N, m}(q) e_{m}(x) F_{N-m}(q, x), \\
A_{N, m}(q)= & \sum_{j=1}^{m} \sum_{\substack{1 \leq k_{i} \leq n \\
k_{1}+\cdots+k_{j}=m}}(-1)^{j+1} q^{c_{N, m}\left(k_{1}, \ldots, k_{j}\right)}, \\
c_{N, m}\left(k_{1}, \ldots, k_{j}\right)= & \frac{1}{2} N(N+1)-\frac{1}{2}(N-m)(N-m+1) \\
& -\sum_{i=1}^{j}\left(N-m+k_{1}+\cdots+k_{i}\right) \\
= & N(m-j)-\frac{1}{2} m^{2}-\frac{1}{2} m+\sum_{i=1}^{j} i k_{i},
\end{aligned}
$$

where $F_{N}(q, x)=0$ for $N<0$.

In the right hand side of (A.4) we can replace the summation $\sum_{m=1}^{N}$ by $\sum_{m=1}^{n}$ because $e_{m}(x)=0$ for $m>n$. To complete the proof of Theorem A.1, we have only to show that

Lemma A.6. For $1 \leq m \leq \min (n, N)$,

$$
A_{N, m}(q)=(-1)^{m+1} \frac{(q)_{N-1}}{(q)_{N-m}}
$$

Proof. For any $N \geq 1, A_{N, 1}(q)=q^{c_{N, 1}(1)}=1$. Below we show

$$
A_{N, m}(q)=-\left(1-q^{N-1}\right) A_{N-1, m-1}(q)
$$

for $2 \leq m \leq N$. From these, the lemma follows.

Consider

$$
A_{N, m}(q)=\sum_{j=1}^{m} \sum_{\substack{k_{i} \geq 1 \\ k_{1}+\cdots+k_{j}=m}}(-1)^{j+1} q^{c_{N, m}\left(k_{1}, \ldots, k_{j}\right)}
$$

for $2 \leq m \leq N$. Notice that we have dropped the upper inequality $k_{i} \leq n$ in the summation, because it is automatically satisfied under the assumption $m \leq n$. Let

$$
I_{m}=\left\{\left(k_{1}, \ldots, k_{j}\right) \mid j, k_{i} \geq 1, k_{1}+\cdots+k_{j}=m\right\}
$$

be the set of all the ordered partitions of $m$. Then $I_{m}$ is the disjoint union of $I_{m}^{(1)}$ and $I_{m}^{(2)}$, where

$$
\begin{aligned}
& I_{m}^{(1)}=\left\{\left(k_{1}, \ldots, k_{j}, 1\right) \mid\left(k_{1}, \ldots, k_{j}\right) \in I_{m-1}\right\}, \\
& I_{m}^{(2)}=\left\{\left(k_{1}, \ldots, k_{j}+1\right) \mid\left(k_{1}, \ldots, k_{j}\right) \in I_{m-1}\right\} .
\end{aligned}
$$


Let us perform the summation in (A.9) over $I_{m}^{(1)}$ and $I_{m}^{(2)}$, separately. The contribution from $I_{m}^{(1)}$ is

$$
\begin{aligned}
& \sum_{j=1}^{m-1} \sum_{\left(k_{1}, \ldots, k_{j}\right) \in I_{m-1}}(-1)^{j+2} q^{c_{N, m}\left(k_{1}, \ldots, k_{j}, 1\right)} \\
= & -\sum_{j=1}^{m-1} \sum_{\left(k_{1}, \ldots, k_{j}\right) \in I_{m-1}}(-1)^{j+1} q^{c_{N-1, m-1}\left(k_{1}, \ldots, k_{j}\right)}=-A_{N-1, m-1}(q) .
\end{aligned}
$$

The contribution from $I_{m}^{(2)}$ is

$$
\begin{aligned}
& \sum_{j=1}^{m-1} \sum_{\left(k_{1}, \ldots, k_{j}\right) \in I_{m-1}}(-1)^{j+1} q^{c_{N, m}\left(k_{1}, \ldots, k_{j}+1\right)} \\
= & \sum_{j=1}^{m-1} \sum_{\left(k_{1}, \ldots, k_{j}\right) \in I_{m-1}}(-1)^{j+1} q^{c_{N-1, m-1}\left(k_{1}, \ldots, k_{j}\right)+N-1}=q^{N-1} A_{N-1, m-1}(q) .
\end{aligned}
$$

Putting the both together, we get (A.8).

\section{Appendix B. A new COMbinatorial formula for KostKa-Foulkes POLYNOMIALS}

As another corollary of Theorem 7.4, or Theorem A.1, we get a new formula for the Kostka-Foulkes polynomials in terms of the Littlewood-Richardson tableaux of border strips. See [16, 19] for further information of the material discussed here. For a border strip $\kappa=\left\langle m_{1}, \ldots, m_{r}\right\rangle$ we define $t(\kappa)$ as

$$
t(\kappa)=\sum_{i=1}^{r-1}(r-i) m_{i}
$$

Further, for a partition $\lambda$, we denote by $C(\kappa, \lambda)$ the number of the semi-standard tableaux that form lattice permutations (cf. [19]) and are of shape $\kappa$ and content $\lambda$. The number $C(\kappa=\mu / \nu, \lambda)$ has an interpretation as $c_{\nu \lambda}^{\mu}=\operatorname{Mult}_{V_{\lambda}}\left(V_{\mu, \nu}\right)$, i.e., the multiplicity of the irreducible representation $V_{\lambda}$ of $s l_{n}$ in the restriction of the representation $V_{\mu, \nu}$ of $Y\left(s l_{n}\right)$ to $s l_{n}$.

Proposition B.1. Let $\lambda$ be a partition, then

$$
\sum_{\kappa} q^{t(\kappa)} C(\kappa, \lambda)=K_{\lambda,\left(1^{|\lambda|}\right)}(q),
$$

where the summation is taken over all the border strips $\kappa$ of rank $n$ with $|\kappa|=|\lambda|$.

Proof. Theorem A.1 can be rewritten as

$$
\sum_{\substack{\kappa: \text { border strip } \\|\kappa|=N}} q^{t(\kappa)} s_{\kappa}(x)=\sum_{\substack{k_{i} \in \mathbf{Z}_{\geq 0} \\ k_{1}+\cdots+k_{n}=N}} q^{\sum_{i=1}^{n} \frac{1}{2} k_{i}\left(k_{i}-1\right)} \frac{(q)_{N}}{(q)_{k_{1}} \cdots(q)_{k_{n}}} x_{1}^{k_{1}} \cdots x_{n}^{k_{n}} .
$$


On the other hand, the degree- $N$ part of Corollary 6 of [16] reads as

$$
\sum_{\substack{\lambda: \text { Young diagram } \\|\lambda|=N}} K_{\lambda,(1|\lambda|)}(q) s_{\lambda}(x)=\sum_{\substack{k_{i} \in \mathbf{Z}_{\geq 0} \\ k_{1}+\cdots+k_{n}=N}} q^{\sum_{i=1}^{n} \frac{1}{2} k_{i}\left(k_{i}-1\right)} \frac{(q)_{N}}{(q)_{k_{1}} \cdots(q)_{k_{n}}} x_{1}^{k_{1}} \cdots x_{n}^{k_{n}} .
$$

Equate the left hand sides of two equalities, and expand $s_{\kappa}(x)$ as $\sum_{\lambda} C(\kappa, \lambda) s_{\lambda}(x)$, where $|\lambda|=|\kappa|$ in the summation. Using the independence of $s_{\lambda}$ 's, we have (B.1).

Example. Let $\lambda=(3,2,1)$. Then it is known [19 that

$$
K_{(3,2,1),\left(1^{6}\right)}(q)=q^{4}(1+q)^{2}\left(1+q^{2}\right)\left(1+q^{3}\right) .
$$

On the other hand, there exist 14 border strips which give non-zero contribution to the left hand side of (B.1) with the following tableaux:
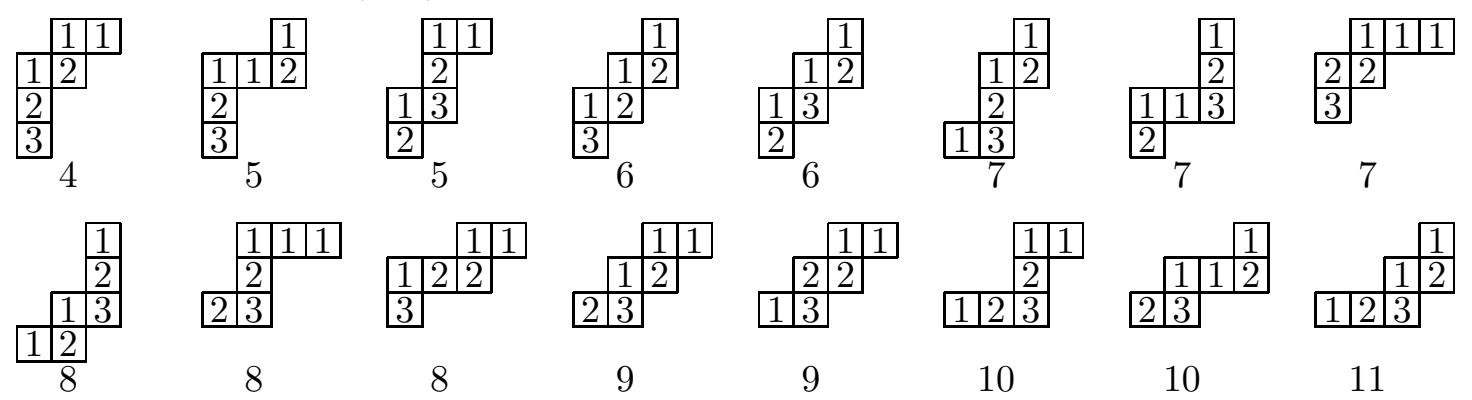

where the number $t(\kappa)$ is attached below each diagram. Hence the left hand side of (B.1) is

$$
q^{4}\left(1+2 q+2 q^{2}+3 q^{3}+3 q^{4}+2 q^{5}+2 q^{6}+q^{7}\right)=K_{(3,2,1),\left(1^{6}\right)}(q) .
$$

In the same spirit we define the branching function $b_{\lambda}^{\Lambda_{k}}(q)$ of $\mathcal{L}\left(\Lambda_{k}\right)$ (as an $s l_{n^{-}}$ module) as

$$
\operatorname{ch} \mathcal{L}\left(\Lambda_{k}\right)(q, x)=\sum_{\substack{\lambda: \text { Young diagram } \\ l(\lambda)<n,|\lambda| \equiv k \bmod n}} b_{\lambda}^{\Lambda_{k}}(q) s_{\lambda}(x) .
$$

Then from (5.4a) we have

\section{Proposition B.2.}

$$
b_{\lambda}^{\Lambda_{k}}(q)=q^{-\frac{c}{24}} \sum_{\substack{\kappa \in B S \\|\kappa| \geq|\lambda|,|\kappa| \equiv k \bmod n}} q^{\frac{1}{2 n}|\kappa|(n-|\kappa|)+t(\kappa)} C\left(\kappa, \lambda+\left(\left(\frac{|\kappa|-|\lambda|}{n}\right)^{n}\right)\right) .
$$

We are going to prove an analogue of (B.1) for the Kostka-Foulkes polynomials $K_{\lambda,\left(\ell^{N}\right)}(q)$ (which should correspond to the $Y\left(s l_{n}\right)$-module structure on $\mathcal{L}\left(\ell \Lambda_{0}\right)$ ) in a separate publication. 
Appendix C. The Character of the level 1 integrable module of $A_{2 n}^{(2)}$

In this appendix we describe the function $\operatorname{ch} \mathcal{L}\left(\Lambda_{n}\right)(q, x)$ in Theorem 8.1.1

Let $\alpha_{0}, \ldots, \alpha_{n}$ be the simple roots of $A_{2 n}^{(2)}$ with the label in Fig. 4 . We have the null $\operatorname{root} \delta=\alpha_{0}+2\left(\alpha_{1}+\cdots \alpha_{n}\right)$. Let $\left\{\epsilon_{1}, \ldots, \epsilon_{n}\right\}$ be the orthonormal basis of the dual of the Cartan subalgebra of $B_{n}$ with

$$
\alpha_{0}=\delta-2 \epsilon_{1}, \quad \alpha_{1}=\epsilon_{1}-\epsilon_{2}, \quad \ldots, \quad \alpha_{n-1}=\epsilon_{n-1}-\epsilon_{n}, \quad \alpha_{n}=\epsilon_{n} .
$$

On the other hand, let $\alpha_{i}^{\prime}=\alpha_{n-i}$, which favors the subalgebra $C_{n}$. (Below the prime symbol ' indicates that we are in the $C_{n}$ picture.) Let $\left\{\epsilon_{1}^{\prime}, \ldots, \epsilon_{n}^{\prime}\right\}$ be the orthonormal basis of the dual of the Cartan subalgebra of $C_{n}$ with

$$
\alpha_{0}^{\prime}=\frac{1}{2} \delta-\epsilon_{1}^{\prime}, \quad \alpha_{1}^{\prime}=\epsilon_{1}^{\prime}-\epsilon_{2}^{\prime}, \quad \ldots, \quad \alpha_{n-1}^{\prime}=\epsilon_{n-1}^{\prime}-\epsilon_{n}^{\prime}, \quad \alpha_{n}^{\prime}=2 \epsilon_{n}^{\prime} .
$$

Comparing (C.1) and (C.2), we have the relation $\epsilon_{i}+\epsilon_{n+1-i}^{\prime}=\frac{1}{2} \delta$.

The unnormalized character of $\mathcal{L}\left(\Lambda_{0}^{\prime}\right)$ is given by [14]

$$
\operatorname{ch} \mathcal{L}\left(\Lambda_{0}^{\prime}\right)=\Theta / \prod_{j=1}^{\infty}\left(1-e^{-j \delta}\right)^{n}, \quad \Theta=\sum_{\gamma^{\prime} \in M^{\prime}} e^{\Lambda_{0}^{\prime}+\gamma^{\prime}-\frac{1}{2}\left|\gamma^{\prime}\right|^{2} \delta}, \quad M^{\prime}=\bigoplus_{i=1}^{n} \mathbf{Z} \epsilon_{i}^{\prime} .
$$

The fundamental weight $\Lambda_{0}^{\prime}$ in the $C_{n}$ picture is related to $\Lambda_{n}$ in the $B_{n}$ picture as $\Lambda_{0}^{\prime}=\Lambda_{n}+b_{0} \delta$ with a certain constant $b_{0}$. Thus $e^{-b_{0} \delta} \operatorname{ch} \mathcal{L}\left(\Lambda_{0}^{\prime}\right)$ is the unnormalized character of $\mathcal{L}\left(\Lambda_{n}\right)$. After the substitution of $\Lambda_{0}^{\prime}=\bar{\Lambda}_{n}+\frac{1}{2} \Lambda_{0}+b_{0} \delta$ and $\epsilon_{i}^{\prime}=\frac{1}{2} \delta-\epsilon_{n+1-i}$, $e^{-b_{0} \delta} \Theta$ is expressed as

$$
e^{-b_{0} \delta} \Theta=e^{\frac{1}{2}\left|\bar{\Lambda}_{n}\right|^{2} \delta} \sum_{\gamma \in M} e^{\frac{1}{2} \Lambda_{0}+\bar{\Lambda}_{n}+\gamma-\frac{1}{2}\left|\bar{\Lambda}_{n}+\gamma\right|^{2} \delta}, \quad M=\bigoplus_{i=1}^{n} \mathbf{Z} \epsilon_{i},
$$

where $\bar{\Lambda}_{n}=\frac{1}{2}\left(\epsilon_{1}+\cdots+\epsilon_{n}\right)$. Finally, by setting $e^{-\delta}=q$ and $e^{\Lambda_{0}}=1$, we have

$$
\operatorname{ch} \mathcal{L}\left(\Lambda_{n}\right)(q, x)=\frac{q^{-\frac{1}{2}\left|\bar{\Lambda}_{n}\right|^{2}}}{(q)_{\infty}^{n}} \sum_{\gamma \in M} q^{\frac{1}{2}\left|\bar{\Lambda}_{n}+\gamma\right|^{2}} e^{\bar{\Lambda}_{n}+\gamma}, \quad e^{\epsilon_{i}}=x_{i},
$$

which is the left hand side of $(8.3)$.

\section{REFERENCES}

[1] G. E. Andrews, The theory of partitions, Addison-Wesley Publ. 1976.

[2] T. Arakawa, T. Nakanishi, K. Oshima, and A. Tsuchiya, Spectral decomposition of path space in solvable lattice model, q-alg/9507025, to appear in Commun. Math. Phys.

[3] R. J. Baxter, Exactly solved models in statistical mechanics, Academic Press, 1982.

[4] D. Bernard, Talk given at Yukawa Institute of Theoretical Physics, Kyoto University, Nov. 1994.

\footnotetext{
${ }^{4}$ It is our pleasure to thank M. Wakimoto for his helpful comments.
} 
[5] D. Bernard, M. Gaudin, F. D. M. Haldane, and V. Pasquier, Yang-Baxter equation in long-range interacting systems, J. Phys. A26 (1993) 5219-5236.

[6] D. Bernard, V. Pasquier, and D. Serban, Spinons in conformal field theory, Nucl. Phys. B428 (1994) 612-628.

[7] I. V. Cherednik, A new interpretation of Gelfand-Zetlin bases, Duke Math. J. 54 (1987) 563-577.

[8] E. Date, M. Jimbo, A. Kuniba, T. Miwa, and M. Okado, Path, Maya diagrams and representation of $s l(r, \mathbf{C})$, Adv. Stud. in Pure Math. 19 (1989) 149-191.

[9] V. G. Drinfel'd, A new realization of Yangians and quantized affine algebras, Soviet Math. Dokl. 36 (1988) 212-216.

[10] Z. N. C. Ha and F. D. M. Haldane, Squeezed strings and Yangian symmetry of the Heisenberg chain with long-range interaction, Phys. Rev. B47 (1993) 12459-12469.

[11] F. D. M. Haldane, Z. N. C. Ha, J. C. Testra, D. Bernard, and V. Pasquier, Yangian symmetry of integrable quantum chains with long-range interactions and a new description of states in conformal field theory, Phys. Rev. Lett. 69 (1992) 2021.

[12] K. Hikami, Yangian symmetry and Virasoro character in a lattice spin system with long-range interactions, Nucl. Phys. B441 [FS] (1995) 530-548.

[13] V. G. Kac, Infinite dimensional Lie algebras, third edition, Cambridge University Press, 1990.

[14] V. G. Kac and D. H. Peterson, Infinite-dimensional Lie algebras, theta functions and modular forms, Advances in Math. 53 (1984) 125-264.

[15] S-J. Kang, M. Kashiwara, K. Misra, T. Miwa, T. Nakashima, A. Nakayashiki, Affine crystals and vertex models, Int. J. Mod. Phys. A7, Suppl. 1A (1992) 449-484.

[16] A. N. Kirillov. Dilogarithm identities, Lectures in Mathematical Sciences 7, University of Tokyo, 1995; Prog. Theor. Phys. (Suppl. 118) (1995) 61-142.

[17] A. Kuniba, Exact solution of solid-on-solid models for twisted affine Lie algebras $A_{2 n}^{(2)}$ and $A_{2 n-1}^{(2)}$, Nucl. Phys. B355 (1991) 801-821.

[18] A. Kuniba, Y. Ohta, J. Suzuki, Quantum Jacobi-Trudi and Giambelli formulae for $U_{q}\left(B_{r}^{(1)}\right)$ from the analytic Bethe ansatz, J. Phys. A: Math. Gen. 28 (1995) 6211-6226.

[19] I. G. Macdonald, Symmetric Functions and Hall Polynomials, second edition 1995, Oxford Univ. Press.

[20] M. Nazarov and V. Tarasov, Representations of Yangians with Gelfand-Zetlin bases, preprint, 1994.

[21] A. P. Polychronakos, Exact spectrum of $S U(n)$ spin chain with inverse-square exchange, Nucl. Phys. B419 [FS] (1994) 553-566.

[22] K. Schoutens, Yangian symmetry in conformal field theory, Phys. Lett. B331 (1994) 335-341. 